\title{
Foundations of a Multi-way Spectral Clustering Framework for Hybrid Linear Modeling *
}

\author{
Guangliang Chen, Gilad Lerman ${ }^{\dagger}$ \\ Department of Mathematics, University of Minnesota \\ 127 Vincent Hall, 206 Church Street SE, Minneapolis, MN 55455 \\ \{chen0796, lerman\}@umn.edu
}

October 31, 2018

\begin{abstract}
The problem of Hybrid Linear Modeling (HLM) is to model and segment data using a mixture of affine subspaces. Different strategies have been proposed to solve this problem, however, rigorous analysis justifying their performance is missing. This paper suggests the Theoretical Spectral Curvature Clustering (TSCC) algorithm for solving the HLM problem, and provides careful analysis to justify it. The TSCC algorithm is practically a combination of Govindu's multi-way spectral clustering framework (CVPR 2005) and Ng et al.'s spectral clustering algorithm (NIPS 2001). The main result of this paper states that if the given data is sampled from a mixture of distributions concentrated around affine subspaces, then with high sampling probability the TSCC algorithm segments well the different underlying clusters. The goodness of clustering depends on the withincluster errors, the between-clusters interaction, and a tuning parameter applied by TSCC. The proof also provides new insights for the analysis of $\mathrm{Ng}$ et al. (NIPS 2001).
\end{abstract}

AMS Subject Classification (2000): 68Q32, 68T05, 62H30 (secondary: 68W40, $60-99,15 \mathrm{~A} 42)$

Keywords: Hybrid linear modeling, clustering $d$-flats, multi-way clustering, spectral clustering, polar curvature, perturbation analysis, concentration inequalities

\section{Introduction}

The problem of Hybrid Linear Modeling (HLM) is to model data using a collection of affine subspaces, or equivalently, flats, and simultaneously segment data into subsets representing those flats (see also formulations in [39] and [26]). This problem has diverse applications in many areas, such as motion segmentation in computer vision, hybrid linear representation of images, classification of face

\footnotetext{
${ }^{*}$ This work was supported by NSF grant \#0612608

${ }^{\dagger}$ Corresponding Author: Gilad Lerman, phone: (612) 624-5541, fax: (612) 626-2017
} 
images, and temporal segmentation of video sequences (see 26 and references therein). Also, it is closely related to sparse representation and manifold learning 35,28 .

Many algorithms and strategies can be applied to this problem. For example, RANSAC [9, 37, 42], $K$-Subspaces [16, 14]/ $K$-Planes [5, 38], Subspace Separation [8, 17, 18, Mixtures of Probabilistic PCA [36], Independent Component Analysis [15, Tensor Voting [30, Multi-way Clustering [2, 11, 32, 1], Generalized Principal Component Analysis 39, 26, Manifold Clustering 34, Local Subspace Affinity [4], Grassmann Clustering [12, Algebraic Multigrid [19], Agglomerative Lossy Compression [25] and Poisson Mixture Model [13. However, we are not aware of any probabilistic analysis of the performance of such algorithms given data sampled from a corresponding hybrid model (with additive noise). The goal of this paper is to rigorously justify a particular solution to the HLM problem.

For simplicity we restrict the discussion to the case where all the underlying flats have the same dimension $d \geq 0$, although our theory extends to mixed dimensions by considering only the maximum dimension. We also assume here that the intrinsic dimension, $d$, and the number of clusters, $K$, are known, and leave their estimation to future works.

Our solution to HLM, the Theoretical Spectral Curvature Clustering (TSCC) algorithm, follows the multi-way spectral clustering framework of Govindu [11. This framework (when applied to HLM) starts by computing an affinity measure quantifying $d$-dimensional flatness for any $d+2$ points of the data. It then forms pairwise weights by decomposing the corresponding $(d+2)$-way affinity tensor. At last, it applies spectral clustering (e.g., [33]) with the pairwise weights. However, these steps are based on heuristic arguments [11, with no formal justification for them.

The TSCC algorithm combines Govindu's framework 11 with Ng et al.'s spectral clustering algorithm [31, while introducing "the polar tensor" (see Subsection 2.4). We justify the TSCC algorithm following the strategy of 31 in two steps. First, we consider a general affinity tensor instead of the polar tensor, and control the goodness of clustering of TSCC by the deviation of the affinity tensor from an ideal tensor (Section 4). Next, we show that for a more restricted class of affinity tensors (also including the polar tensor) and data sampled from a hybrid linear model, the TSCC algorithm clusters the data well with high sampling probability (Section 5.2). For the polar tensor, the goodness of clustering can be expressed in terms of the within-cluster errors (which depend directly on the flatness of the underlying measures), the betweenclusters interaction (which depends on the separation of the measures), and a tuning parameter applied by TSCC (Section 5).

The rest of the paper is organized as follows. In Section 2 we review some theoretical background. In Section 3 we present the TSCC algorithm as a combination of Govindu's framework [11] and Ng et al.'s algorithm 31] while using the specific polar tensor. Both Sections 4 and 5 analyze the performance of the TSCC algorithm. The former section presents the main technical estimates for a large class of affinity tensors, while quantifying fundamental notions, in particular, the goodness of clustering. The latter section assumes a hybrid linear probabilistic model and the use of the polar tensor, and relates the estimates of Section 4 to the sampling distribution of the model. Section 6 concludes with a brief discussion and possible avenues for future work. Mathematical proofs are 
given in the appendix.

\section{Background}

\subsection{Notation and Basic Definitions}

Throughout this paper we assume an ambient space $\mathbb{R}^{D}$ and a collection of $d$-flats that are embedded in $\mathbb{R}^{D}$, where $0 \leq d<D$.

We denote scalars with possibly large values by upper-case plain letters (e.g., $N, C$ ), and scalars with relatively small values by lower-case Greek letters (e.g., $\alpha, \varepsilon)$; vectors by boldface lower-case letters (e.g., $\mathbf{u}, \mathbf{v})$; matrices by boldface upper-case letters (e.g., A); tensors by calligraphic capital letters (e.g., $\mathcal{A}$ ); and sets by upper-case Roman letters (e.g., X).

For any integer $n>0$, we denote the $n$-dimensional vector of ones by $\mathbf{1}_{n}$, and the $n \times n$ matrix of ones by $\mathbf{1}_{n \times n}$. The $n \times n$ identity matrix is written as $\mathbf{I}_{n}$.

The $(i, j)$-element of a matrix $\mathbf{A}$ is denoted by $A_{i j}$, and the $\left(i_{1}, \ldots, i_{n}\right)$ element of an $n$-way tensor $\mathcal{A}$ is denoted by $\mathcal{A}\left(i_{1}, \ldots, i_{n}\right)$. We denote the transpose of a matrix $\mathbf{A}$ by $\mathbf{A}^{\prime}$ and that of a vector $\mathbf{v}$ by $\mathbf{v}^{\prime}$. The Frobenius norm of a matrix/tensor, denoted by $\|\cdot\|_{F}$, is the $\ell_{2}$ norm of the quantity when viewed as a vector.

If $k>0$ is an integer and $\mathbf{A}$ is a positive semidefinite square matrix, we use $E_{k}(\mathbf{A})$ to denote the subspace spanned by the top $k$ eigenvectors of $\mathbf{A}$, and $P^{k}(\mathbf{A})$ to represent the orthogonal projector onto $E_{k}(\mathbf{A})$.

If $\mathbf{x} \in \mathbb{R}^{D}$ and $F$ is a $d$-flat in $\mathbb{R}^{D}$, then we denote the orthogonal distance from $\mathbf{x}$ to $F$ by $\operatorname{dist}(\mathbf{x}, F)$. For any $r>0$, the ball centered at $\mathbf{x}$ with radius $r$ is written as $\mathrm{B}(\mathbf{x}, r)$. If $c>0$, then $c \cdot \mathrm{B}(\mathbf{x}, r):=\mathrm{B}(\mathbf{x}, c \cdot r)$. If $\mathrm{S}$ is a subset of $\mathbb{R}^{D}$, we denote its diameter by $\operatorname{diam}(\mathrm{S})$ and its complement by $\mathrm{S}^{c}$. If $\mathrm{S}$ is furthermore discrete, we use $|S|$ to denote its number of elements.

Let $\mu$ be a measure on $\mathbb{R}^{D}$. We denote the support of $\mu$ by $\operatorname{supp}(\mu)$, its restriction to a given set $\mathrm{S}$ by $\left.\mu\right|_{\mathrm{S}}$, and the product measure of $n$ copies of $\mu$, where $n \in \mathbb{N}$, by $\mu^{n}$. The $d$-dimensional Lebesgue measure is denoted by $\mathcal{L}_{d}$. Also, we use $\left(\mathbb{R}^{D}\right)^{n}$ to denote the Cartesian product of $n$ copies of $\mathbb{R}^{D}$.

We use $\mathrm{P}(n, r)$ to denote the number of permutations of size $r$ from a sequence of $n$ available elements. That is,

$$
\mathrm{P}(n, r):=n(n-1) \cdots(n-r+1) .
$$

\subsection{The Problem of Hybrid Linear Modeling}

We formulate here a version of the problem of HLM. We will introduce further restrictions on its setting throughout the paper. Before presenting the problem we need to define the notions of $d$-dimensional least squares errors and flats.

If $\mu$ is a Borel probability measure, then the least squares error of approximating $\mu$ by a $d$-flat is denoted by $e_{2}(\mu)$ and defined as follows:

$$
e_{2}(\mu):=\sqrt{\inf _{d \text {-flats } F} \int \operatorname{dist}^{2}(\mathbf{x}, F) \mathrm{d} \mu(\mathbf{x})} .
$$

Any minimizer of the above quantity is referred to as a least squares $d$-flat. 
We now incorporate the above definitions and present the problem of hybrid linear modeling below.

Problem 1. Let $\mu_{1}, \ldots, \mu_{K}$ be Borel probability measures and assume that their $d$-dimensional least square errors $\left\{e_{2}\left(\mu_{k}\right)\right\}_{k=1}^{K}$ are sufficiently small and that their least squares d-flats do not coincide. Suppose a data set $\mathrm{X}=\left\{\mathbf{x}_{1}, \ldots, \mathbf{x}_{N}\right\} \subset$ $\mathbb{R}^{D}$ generated as follows: For each $k=1, \ldots, K, N_{k}$ points are sampled independently and identically from $\mu_{k}$, so that $N=N_{1}+\cdots+N_{K}$. The goal of hybrid linear modeling is to segment $\mathrm{X}$ into $K$ subsets representing the underlying $d$-flats and simultaneously estimate the parameters of the underlying flats.

We remark that the above notion of sufficiently small least square errors combined with non-coinciding least squares $d$-flats is quantified for our particular solution later in Subsection 5.2 (by restricting the size of the constant $\alpha$ of equation (33)). We also remark that we restrict the above setting in Subsection 2.3 by requiring the measures $\mu_{1}, \ldots, \mu_{K}$ to be regular and possibly $d$-separated (see Remark 2.5) and later in Subsection 4.2.1 by imposing the comparability of sizes of $N_{1}, \ldots, N_{K}$ (see equation (12)).

\subsection{The Polar Curvature}

For any $d+2$ distinct points $\mathbf{z}_{1}, \ldots, \mathbf{z}_{d+2} \in \mathbb{R}^{D}$, we denote by $V_{d+1}\left(\mathbf{z}_{1}, \ldots, \mathbf{z}_{d+2}\right)$ the $(d+1)$-volume of the $(d+1)$-simplex formed by these points. The polar sine at each vertex $\mathbf{z}_{i}, 1 \leq i \leq d+2$, is

$$
\operatorname{psin}_{\mathbf{z}_{i}}\left(\mathbf{z}_{1}, \ldots, \mathbf{z}_{d+2}\right):=\frac{(d+1) ! \cdot V_{d+1}\left(\mathbf{z}_{1}, \ldots, \mathbf{z}_{d+2}\right)}{\prod_{1 \leq j \leq d+2, j \neq i}\left\|\mathbf{z}_{j}-\mathbf{z}_{i}\right\|_{2}} .
$$

Definition 2.1. The polar curvature of $\mathbf{z}_{1}, \ldots, \mathbf{z}_{d+2}$ is

$$
c_{\mathrm{p}}\left(\mathbf{z}_{1}, \ldots, \mathbf{z}_{d+2}\right):=\operatorname{diam}\left(\left\{\mathbf{z}_{1}, \ldots, \mathbf{z}_{d+2}\right\}\right) \cdot \sqrt{\sum_{i=1}^{d+2} \operatorname{psin}_{\mathbf{z}_{i}}^{2}\left(\mathbf{z}_{1}, \ldots, \mathbf{z}_{d+2}\right)} .
$$

Remark 2.2. The notion of curvature designates here a function of $d+2$ variables generalizing the distance function. Indeed, when $d=0$, the polar curvature coincides with the Euclidean distance. We use this name (and probably abuse it) due to the comparability when $d=1$ of the polar curvature with the Menger curvature multiplied by the square of the corresponding diameter (see [21]).

Let $\mu$ be a Borel probability measure on $\mathbb{R}^{D}$. We define the polar curvature of $\mu$ to be

$$
c_{\mathrm{p}}(\mu):=\sqrt{\int c_{\mathrm{p}}^{2}\left(\mathbf{z}_{1}, \ldots, \mathbf{z}_{d+2}\right) \mathrm{d} \mu\left(\mathbf{z}_{1}\right) \ldots \mathrm{d} \mu\left(\mathbf{z}_{d+2}\right)} .
$$

The polar curvatures of randomly sampled $(d+1)$-simplices can be used to estimate the least squares errors of approximating certain probability measures by $d$-flats. We start with two preliminary definitions and then state the main result, which is proved in [23] (following the methods of [24, 21, 22]). 
Definition 2.3. We say that a Borel probability measure $\mu$ on $\mathbb{R}^{D}$ is $d$-separated (with parameters $0<\delta, \omega<1$ ) if there exist $d+2$ balls $\left\{B_{i}\right\}_{i=1}^{d+2}$ in $\mathbb{R}^{D}$ with $\mu$-measures at least $\delta$ such that

$$
V_{d}\left(\mathbf{x}_{i_{1}}, \ldots, \mathbf{x}_{i_{d+1}}\right)>\omega \cdot \operatorname{diam}(\operatorname{supp}(\mu))^{d},
$$

for any $\mathbf{x}_{i_{k}} \in 2 B_{i_{k}}, 1 \leq k \leq d+1$ and $1 \leq i_{1}<\cdots<i_{d+1} \leq d+2$.

Definition 2.4. We say that a Borel probability measure $\mu$ on $\mathbb{R}^{D}$ is regular (with parameters $C_{\mu}$ and $\gamma$ ) if there exist constants $\gamma>2$ and $C_{\mu} \geq 1$ such that for any $\mathbf{x} \in \operatorname{supp}(\mu)$ and $0<r \leq \operatorname{diam}(\operatorname{supp}(\mu))$ :

$$
\mu(B(\mathbf{x}, r)) \leq C_{\mu} r^{\gamma}
$$

If $D=2(\operatorname{or} \operatorname{supp}(\mu)$ is contained in a 2-flat), then one can allow $1<\gamma \leq 2$ while strengthening the above equation as follows:

$$
C_{\mu}^{-1} r^{\gamma} \leq \mu(B(\mathbf{x}, r)) \leq C_{\mu} r^{\gamma}
$$

Theorem 2.1. For any regular and $d$-separated Borel probability measure $\mu$ there exists a constant $C$ (depending only on the d-separation parameters, i.e., $\omega, \delta$, and the regularity parameters, i.e., $\gamma, C_{\mu}$ ) such that

$$
C^{-1} \cdot e_{2}(\mu) \leq c_{\mathrm{p}}(\mu) \leq C \cdot e_{2}(\mu)
$$

The following two curvatures also satisfy Theorem 2.1$] 23]$

$$
\begin{aligned}
c_{\mathrm{dls}}\left(\mathbf{z}_{1}, \ldots, \mathbf{z}_{d+2}\right) & :=\sqrt{\inf _{d-\text { flats } F} \sum_{1 \leq i \leq d+2} \operatorname{dist}^{2}\left(\mathbf{z}_{i}, F\right)}, \\
c_{\mathrm{h}}\left(\mathbf{z}_{1}, \ldots, \mathbf{z}_{d+2}\right) & :=\min _{1 \leq i \leq d+2} \operatorname{dist}\left(\mathbf{z}_{i}, F_{(i)}\right),
\end{aligned}
$$

where $F_{(i)}$ is the $(d-1)$-flat spanned by all the $d+2$ points except $\mathbf{z}_{i}$. In this paper we use $c_{\mathrm{p}}$ as a representative of the class of curvatures that satisfy Theorem 2.1. since it seems computationally faster than the above two (using the numerical framework described in [7]). However, all the theory developed in this paper applies to the rest of the class.

Remark 2.5. Since we use Theorem 2.1 in Subsection 5.3 to justify our proposed solution for HLM, we need to assume that the measures $\mu_{1}, \ldots, \mu_{K}$ of Problem 1 are regular and $d$-separated. However, those restrictions could be relaxed or avoided as follows. If either $c_{\mathrm{dls}}$ or $c_{\mathrm{h}}$ is used instead of $c_{\mathrm{p}}$, then Theorem 2.1 holds for mere $d$-separated probability measures (no need for regularity). Moreover, in Subsection 5.3 we may only use the right hand side of equation (5), i.e., the bound of $c_{\mathrm{p}}(\mu)$ in terms of $e_{2}(\mu)$ (though it is preferable to have a tight estimate as suggested by the full equation). For such a bound it is enough to assume that $\mu$ is merely a regular probability measure. If we use instead of $c_{\mathrm{p}}$ any of the curvatures $c_{\mathrm{dls}}, c_{\mathrm{h}}$, then this latter bound holds for any Borel probability measure. We also comment that the regularity conditions described in Definition 2.4 could be further relaxed when replacing $\operatorname{diam}\left(\left\{\mathbf{z}_{1}, \ldots, \mathbf{z}_{d+2}\right\}\right)$ in equation (3) with e.g., a geometric mean of corresponding edge lengths. More details appear in [23]. 


\subsection{Affinity Tensors and their Matrix Representations}

Throughout the rest of this paper, we consider $(d+2)$-way tensors of the form

$$
\left\{\mathcal{A}\left(i_{1}, \ldots, i_{d+2}\right)\right\}_{1 \leq i_{1}, \ldots, i_{d+2} \leq N} .
$$

We assume that their elements are between zero and one, and invariant under arbitrary permutations of the indices $\left\{i_{1}, \ldots, i_{d+2}\right\}$, i.e., these tensors are supersymmetric.

Most commonly, we form the following affinities using the polar curvature:

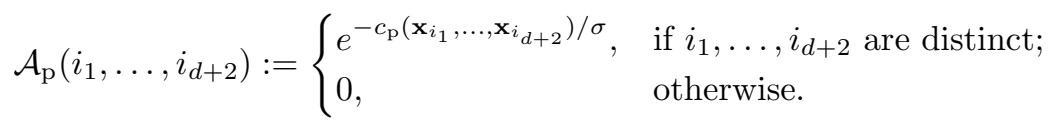

The corresponding tensor $\mathcal{A}_{\mathrm{p}}$ is referred to as the polar tensor.

In the special case of underlying linear subspaces (instead of general affine ones), we may work with the following $(d+1)$-tensor:

$$
\mathcal{A}_{\mathrm{p}, \mathrm{L}}\left(i_{1}, \ldots, i_{d+1}\right):= \begin{cases}e^{-c_{\mathrm{p}}\left(\mathbf{0}, \mathbf{x}_{i_{1}}, \ldots, \mathbf{x}_{i_{d+1}}\right) / \sigma}, & \text { if } i_{1}, \ldots, i_{d+1} \text { are distinct; } \\ 0, & \text { otherwise. }\end{cases}
$$

In most of the paper we use the $(d+2)$-tensor $\mathcal{A}_{\mathrm{p}}$, while in a few places we refer to the $(d+1)$-tensor $\mathcal{A}_{\mathrm{p}, \mathrm{L}}$.

Given a $(d+2)$-way affinity tensor $\mathcal{A} \in \mathbb{R}^{N \times N \times \cdots \times N}$ we unfold it into an $N \times N^{d+1}$ matrix $\mathbf{A}$ in a similar way as in 4,20 . The $i^{\text {th }}$ row of $\mathbf{A}$ contains all the elements in the $i^{\text {th }}$ "slice" of $\mathcal{A}:\left\{\mathcal{A}\left(i, i_{2}, \ldots, i_{d+2}\right), 1 \leq i_{2}, \ldots, i_{d+2} \leq N\right\}$, according to an arbitrary but fixed ordering of the last $d+1$ indices $\left(i_{2}, \ldots, i_{d+2}\right)$, e.g., the lexicographic ordering. This ordering (when fixed for all rows) is not important to us, since we are only interested in the uniquely determined matrix $\mathbf{W}:=\mathbf{A A}^{\prime}$ (see Algorithm 1 below).

\section{Theoretical Spectral Curvature Clustering}

We combine Govindu's framework of multi-way spectral clustering [1] with Ng et al.'s spectral clustering algorithm 31, while incorporating the polar affinities (equation (6)), to formulate below (Algorithm 1) the Theoretical Spectral Curvature Clustering (TSCC) algorithm for solving Problem 1.

We refer to this algorithm as theoretical because its complexity and storage requirement can be rather large (even though polynomial). In 7] we make the algorithm practical by applying various numerical techniques. In particular, we suggest a sampling strategy to approximate the matrix $\mathbf{W}$ in an iterative way, an automatic scheme of tuning the parameter $\sigma$, and a straightforward procedure to initialize $K$-means for clustering the rows of $\mathbf{U}$.

The TSCC algorithm can be seen as two steps of embedding data followed by $K$-means. First, each data point $\mathbf{x}_{i}$ is mapped to $\mathbf{A}(i,:)$, the $i^{\text {th }}$ row of the matrix $\mathbf{A}$, which contains the interactions between the point $\mathbf{x}_{i}$ and all $d$-flats spanned by any $d+1$ points in the data (indeed, each column corresponds to $d+1$ data points). Second, $\mathbf{x}_{i}$ is further mapped to the $i^{\text {th }}$ row of the matrix $\mathbf{U}$. The rows of $\mathbf{U}$ are treated as points in $\mathbb{R}^{K}$, to which $K$-means is applied.

The question of whether or not to normalize the rows of the matrix $\mathbf{U}$ is an interesting one. For ease of the subsequent theoretical development in this 


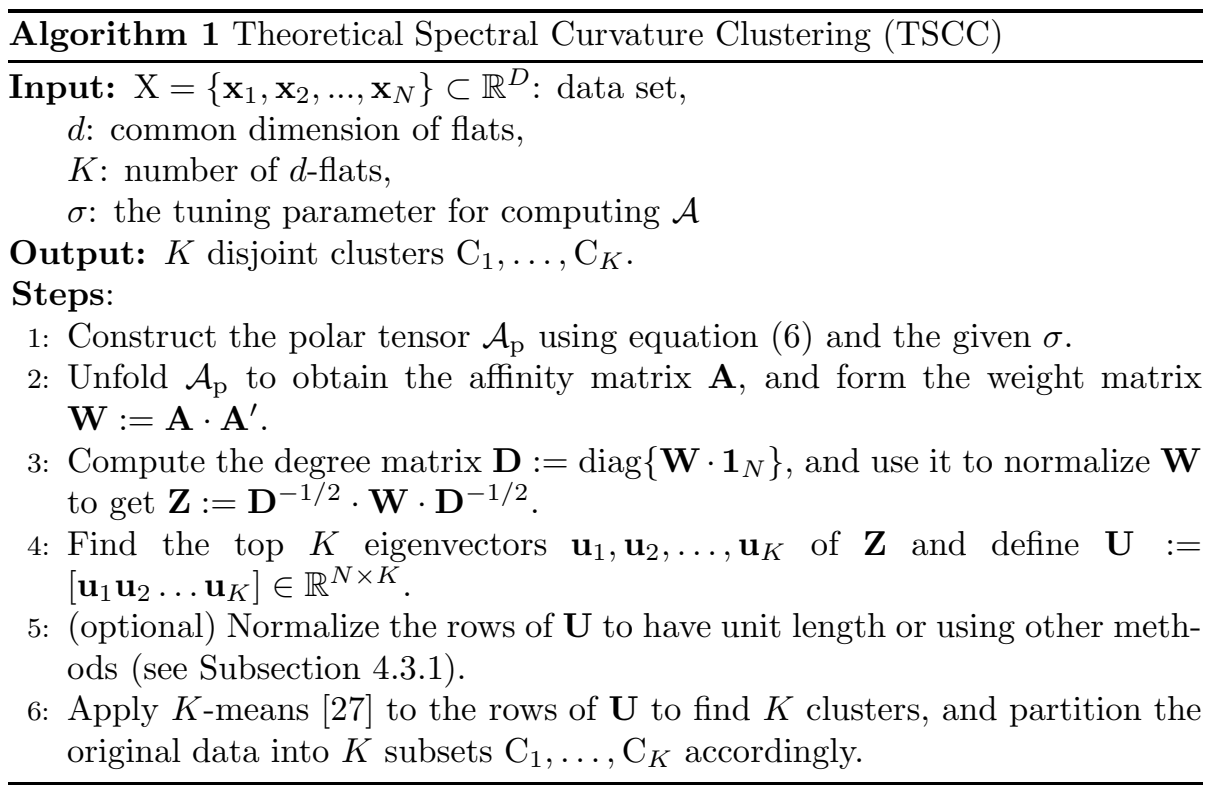

paper we do not normalize the rows of $\mathbf{U}$. Such a choice was also adopted in 7 where the TSCC algorithm yielded good numerical results. In Subsection 4.3.1 we discuss more carefully the normalization of the matrix $\mathbf{U}$ and show the advantage of such practice.

We remark that one can replace the polar tensor (applied in Step 1 of Algorithm 11) with other affinity tensors, based on the polar curvature or other ones that satisfy Theorem 2.1, to form different versions of TSCC. For example, when the underlying subspaces are known to be linear, one may use the $(d+1)$-tensor $\mathcal{A}_{\mathrm{p}, \mathrm{L}}$ of equation (7), forming the Theoretical Linear Spectral Curvature Clustering (TLSCC) algorithm. Another example is the following class of affinity tensors that are based on the powers of the polar curvature:

$$
\mathcal{A}_{\mathrm{p}, q}\left(i_{1}, \ldots, i_{d+2}\right):= \begin{cases}e^{\left.-\frac{c_{\mathrm{p}}^{q}\left(\mathbf{x}_{i_{1}}, \ldots, \mathbf{x}_{i}\right.}{\sigma}\right)}, & \text { if } i_{1}, \ldots, i_{d+2} \text { are distinct } \\ 0, & \text { otherwise }\end{cases}
$$

where $q \geq 1$ (see Remark 5.4 for interpretation). While Algorithm 1 uses $q=1$, its formulation in [7] uses $q=2$, as the latter version of TSCC, when applied in an iterative way, converges faster.

We justify the TSCC algorithm in two steps. In Section 4 we analyze the TSCC algorithm with a very general tensor (replacing the polar tensor), and develop conditions under which TSCC is expected to work well. In particular, the corresponding analysis applies to the polar tensor. In Section 5 we relate this analysis with the sampling of Problem 1 and correspondingly formulate a probabilistic statement for TSCC. The use of the polar curvature yields a clear explanation for the statement. 


\section{Analysis of TSCC with a General Affinity Ten- sor}

Following a strategy of $\mathrm{Ng}$ et al. 31, we analyze the performance of the TSCC algorithm with a general affinity tensor (replacing the polar tensor in Step 1 of Algorithm 10 in two steps. First, we define a "perfect" tensor representing the ideal affinities, and show that in such a hypothetical situation, the $K$ underlying clusters are correctly separated by the TSCC algorithm. Next, we assume that TSCC is applied with a general affinity tensor, and control the goodness of clustering of TSCC by the deviation of the given tensor from the perfect tensor. Finally, we discuss the effect of the two normalizations in the TSCC algorithm (Steps 3 and 5 of Algorithm 1).

\section{Notational Convenience}

We maintain the common setting of Problem 1 and all the notation used in the TSCC algorithm.

We denote the $K$ underlying clusters by $\widetilde{\mathrm{C}}_{1}, \ldots, \widetilde{\mathrm{C}}_{K}$. Each $\widetilde{\mathrm{C}}_{k}$ has $N_{k}$ points, so that $N=\sum_{1 \leq k \leq K} N_{k}$. For ease of presentation we suppose that $N_{1} \leq N_{2} \leq$ $\cdots \leq N_{K}$, and that the points in $\mathrm{X}$ are ordered according to their membership. That is, the first $N_{1}$ points of $\mathrm{X}$ are in $\widetilde{\mathrm{C}}_{1}$, the next $N_{2}$ points in $\widetilde{\mathrm{C}}_{2}$, etc..

We define $K$ index sets $\mathrm{I}_{1}, \ldots, \mathrm{I}_{K}$ having the indices of the points in $\widetilde{\mathrm{C}}_{1}, \ldots, \widetilde{\mathrm{C}}_{K}$ respectively, that is,

$$
\mathrm{I}_{k}:=\left\{n \in \mathbb{N} \mid \sum_{1 \leq j \leq k-1} N_{j}<n \leq \sum_{1 \leq j \leq k} N_{j}\right\}, \quad \text { for each } 1 \leq k \leq K .
$$

We let $\mathbf{u}^{(i)}, 1 \leq i \leq N$, denote the $i^{\text {th }}$ row of $\mathbf{U}$ and $\mathbf{c}^{(k)}, 1 \leq k \leq K$, denote the center of the $k^{\text {th }}$ cluster, i.e.,

$$
\mathbf{c}^{(k)}:=\frac{1}{N_{k}} \sum_{j \in \mathrm{I}_{k}} \mathbf{u}^{(j)}
$$

\subsection{Analysis of TSCC with the Perfect Tensor}

We define here the notion of a perfect tensor and show that TSCC obtains a perfect segmentation with such a tensor.

Definition 4.1. The perfect tensor associated with Problem 1 is defined as follows. For any $1 \leq i_{1}, \ldots, i_{d+2} \leq N$,

$$
\widetilde{\mathcal{A}}\left(i_{1}, \ldots, i_{d+2}\right):= \begin{cases}1, & \text { if } \mathbf{x}_{i_{1}}, \ldots, \mathbf{x}_{i_{d+2}} \text { are distinct and in the same } \widetilde{\mathrm{C}}_{k} \\ 0, & \text { otherwise. }\end{cases}
$$

We designate quantities derived from the perfect tensor $\widetilde{\mathcal{A}}$ (by following the TSCC algorithm) with the tilde notation, e.g., $\widetilde{\mathbf{A}}, \widetilde{\mathbf{W}}, \widetilde{\mathbf{D}}, \widetilde{\mathbf{Z}}, \widetilde{\mathbf{U}}$.

Remark 4.2. When $d=0$, the perfect tensor $\widetilde{\mathcal{A}}$ reduces to a block diagonal matrix, with the blocks corresponding to the underlying clusters. $\mathrm{Ng}$ et al. 31. 
also considered an ideal affinity matrix with a block diagonal structure. However, they maintained the diagonal blocks computed from the data, while we assume a more extreme case in which the elements of these blocks are identically one (except at the diagonal entries). With our assumption it is possible to follow the steps of TSCC and exactly compute each quantity.

Our result for TSCC with the perfect tensor $\widetilde{\mathcal{A}}$ is formulated as follows (see proof in Appendix A.1.

Proposition 4.1. If $N_{k}>d+2$ for all $k=1, \ldots, K$, then

1. $\widetilde{\mathbf{Z}}$ has exactly $K$ eigenvalues of one; the rest are $\frac{d+1}{\left(N_{k}-1\right)\left(N_{k}-d-1\right)}, 1 \leq k \leq$ $K$, each replicated $N_{k}-1$ times.

2. The rows of $\tilde{\mathbf{U}}$ are $K$ mutually orthogonal vectors in $\mathbb{R}^{K}$. Moreover, each vector corresponds to a distinct underlying cluster.

Remark 4.3. For the TLSCC algorithm, the corresponding perfect tensor $\widetilde{\mathcal{A}}_{\mathrm{L}}$ is a $(d+1)$-dimensional equivalent of the $(d+2)$-way tensor $\mathcal{A}$ of equation (11). Proposition 4.1 still holds for $\widetilde{\mathcal{A}}_{\mathrm{L}}$ but with $d$ replaced by $d-1$.

Example 4.4. Illustration of the perfect tensor analysis: We randomly generate three clean lines in $\mathbb{R}^{2}$ and then sample 25 points from each line (see Figure 1(a) . We then apply TSCC with the polar tensor of equation (6) and $\sigma=.00001$. The corresponding tensor is a close approximation to the perfect tensor, because taking the limit of equation (6) as $\sigma \rightarrow 0+$ essentially yields the perfect tensor. Intermediate and final clustering results are reported in Figures 1(b) $1(\mathrm{~d})$

In this case, the top three eigenvalues are hardly distinguished from 1 , and the rest are close to zero (see Figure $1(\mathrm{~b})$. The rows of $\mathbf{U}$ accumulate at three orthogonal vectors (see Figure 1(c)], and thus form three tight clusters, each representing an underlying line (see Figure $1(\mathrm{~d})$ ).

\subsection{Perturbation Analysis of TSCC with a General Affin- ity Tensor}

\subsubsection{Assumptions}

We assume that the underlying clusters have comparable and adequate sizes, more precisely, there exists a constant $0<\varepsilon_{1} \leq 1$ such that

$$
N_{k} \geq \max \left(\varepsilon_{1} \cdot N / K, 2 d+3\right), \quad k=1, \ldots, K .
$$

We also assume that all the affinity tensors $\mathcal{A}$ considered in this section are super-symmetric, and with elements between 0 and 1 . Moreover, they satisfy the following condition.

Assumption 1. There exists a constant $\varepsilon_{2}>0$ such that

$$
\mathbf{D} \geq \varepsilon_{2} \cdot \widetilde{\mathbf{D}} .
$$




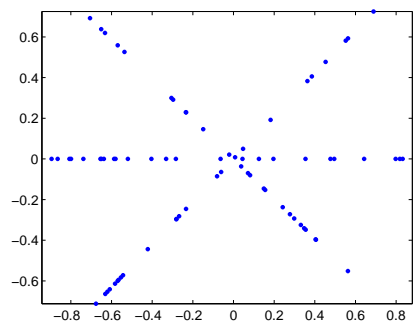

(a) data points

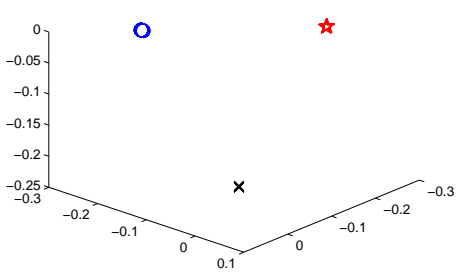

(c) rows of $\mathbf{U}$

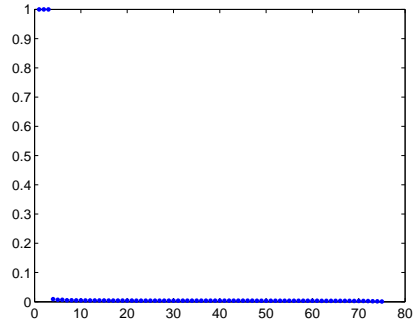

(b) eigenvalues of $\mathbf{Z}$

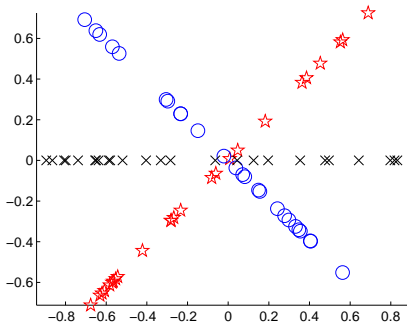

(d) detected clusters

Figure 1: Illustration of the perfect tensor analysis

Remark 4.5. We feel the need to have some lower bound on $\mathbf{D}$, possibly even weaker than that of Assumption 1, to ensure that the TSCC algorithm would work well. Indeed, for each $i \in \mathrm{I}_{k}, 1 \leq k \leq K$, the sum $\sum_{j \in \mathrm{I}_{k}} W_{i j}$ measures the "connectedness" between the point $\mathbf{x}_{i}$ and the other points in $\widetilde{\mathrm{C}}_{k}$, and thus should be sufficiently large. Accordingly, since $D_{i i} \geq \sum_{j \in \mathrm{I}_{k}} W_{i j}, i \in \mathrm{I}_{k}, 1 \leq$ $k \leq K$, these diagonal entries of the matrix $\mathbf{D}$ should be correspondingly large as well. In Subsection 5.4 we discuss the existence of this condition for the polar tensor while taking into account the restrictions on the tuning parameter $\sigma$ implied by Theorem 5.1 .

\subsubsection{Measuring Goodness of Clustering of the TSCC Algorithm}

We use two equivalent ways to quantify the goodness of clustering of the TSCC algorithm when applied with a general affinity tensor $\mathcal{A}$. In Subsection 4.3.1 we relate them to the more absolute notion of clustering identification error.

We first investigate each of the $K$ underlying clusters in the $\mathbf{U}$ space, i.e., $\left\{\mathbf{u}^{(i)}\right\}_{i \in \mathrm{I}_{k}}, 1 \leq k \leq K$, and estimate the sum of their variances. We refer to this sum as the total variation of the matrix $\mathbf{U}$.

Definition 4.6. The total variation of $\mathbf{U}$ (with respect to the $K$ underlying clusters) is

$$
\mathrm{TV}(\mathbf{U}):=\sum_{1 \leq k \leq K} \sum_{i \in \mathrm{I}_{k}}\left\|\mathbf{u}^{(i)}-\mathbf{c}^{(k)}\right\|_{2}^{2},
$$


where $\mathbf{c}^{(1)}, \ldots, \mathbf{c}^{(K)}$ are the centers of the underlying clusters in the $\mathbf{U}$ space (see equation (100)).

The smaller the total variation $\mathrm{TV}(\mathbf{U})$ is, the more concentrated the underlying clusters in the $\mathbf{U}$ space are. In fact, the following lemma (proved in Appendix A.3) implies that the smaller $\mathrm{TV}(\mathbf{U})$ is, the more separated the centers are from the origin and from each other.

\section{Lemma 4.2.}

$$
\begin{gathered}
\sum_{1 \leq k \leq K} N_{k} \cdot\left\|\mathbf{c}^{(k)}\right\|_{2}^{2}=K-\operatorname{TV}(\mathbf{U}), \\
\sum_{1 \leq k<\ell \leq K} N_{k} N_{\ell} \cdot\left\langle\mathbf{c}^{(k)}, \mathbf{c}^{(\ell)}\right\rangle^{2} \leq \mathrm{TV}(\mathbf{U}) .
\end{gathered}
$$

The other measurement of the goodness of clustering of TSCC is motivated by the fact that, in the ideal case, the subspace spanned by the top $K$ eigenvectors of $\widetilde{\mathbf{Z}}, E_{K}(\widetilde{\mathbf{Z}})$, leads to a perfect segmentation (see Proposition 4.1). When given a general affinity tensor $\mathcal{A}$, the eigenspace $E_{K}(\mathbf{Z})$ determines the clustering result of TSCC. We thus suggest to measure the discrepancy between these two eigenspaces, $E_{K}(\mathbf{Z})$ and $E_{K}(\widetilde{\mathbf{Z}})$, by comparing the orthogonal projectors onto them, $P^{K}(\mathbf{Z})$ and $P^{K}(\widetilde{\mathbf{Z}})$, in the following way.

Definition 4.7. The distance between the two subspaces $E_{K}(\widetilde{\mathbf{Z}})$ and $E_{K}(\mathbf{Z})$ is

$$
\operatorname{dist}\left(E_{K}(\mathbf{Z}), E_{K}(\widetilde{\mathbf{Z}})\right):=\left\|P^{K}(\mathbf{Z})-P^{K}(\widetilde{\mathbf{Z}})\right\|_{\mathrm{F}} .
$$

A geometric interpretation of the above distance is provided in the following lemma using the notion of principal angles 10. We review the definition of principal angles and also prove Lemma 4.3 in Appendix A.4

Lemma 4.3. Let $0 \leq \theta_{1} \leq \theta_{2} \leq \cdots \leq \theta_{K} \leq \pi / 2$ be the $K$ principal angles between the two subspaces $E_{K}(\mathbf{Z})$ and $E_{K}(\widetilde{\mathbf{Z}})$. Then

$$
\operatorname{dist}^{2}\left(E_{K}(\mathbf{Z}), E_{K}(\widetilde{\mathbf{Z}})\right)=2 \cdot \sum_{k=1}^{K} \sin ^{2} \theta_{k}
$$

At last, we claim that the above two ways of measuring the goodness of clustering of TSCC are equivalent in the following sense (see proof in Appendix A.2).

Lemma 4.4.

$$
\operatorname{dist}^{2}\left(E_{K}(\mathbf{Z}), E_{K}(\widetilde{\mathbf{Z}})\right)=2 \cdot \operatorname{TV}(\mathbf{U}) .
$$

\subsubsection{The Perturbation Result}

Given a general affinity tensor $\mathcal{A}$ we quantify its deviation from the perfect tensor $\widetilde{\mathcal{A}}$ by the difference

$$
\mathcal{E}:=\mathcal{A}-\widetilde{\mathcal{A}} \text {. }
$$

Our main result shows that the magnitude of this perturbation controls the goodness of clustering of the TSCC algorithm. 
Theorem 4.5. Let $\mathcal{A}$ be any affinity tensor satisfying Assumption 1 and $\mathcal{E}$ its deviation from the perfect tensor. There exists a constant $C_{1}=C_{1}\left(K, d, \varepsilon_{1}, \varepsilon_{2}\right)$ (estimated in equation (71) of Appendix A.5) such that if

$$
N^{-(d+2)}\|\mathcal{E}\|_{\mathrm{F}}^{2} \leq \frac{1}{8 C_{1}}
$$

then

$$
\mathrm{TV}(\mathbf{U}) \leq C_{1} \cdot N^{-(d+2)}\|\mathcal{E}\|_{\mathrm{F}}^{2} .
$$

Remark 4.8. For the TLSCC algorithm, Theorem 4.5 holds with $d$ replaced by $d-1$.

Example 4.9. Illustration of the perturbation analysis: We corrupt the data in Figure1 with 2.5\% additive Gaussian noise (see Figure 2(a)), and apply TSCC with the polar tensor of equation (6) and $\sigma=0.1840$. In this case of moderate noise, the top three eigenvalues are still nicely separated from the rest, even though two of them deviate from 1 (see Figure 2(b)). The rows of $\mathbf{U}$ still form three clear clusters, but they deviate from concentrating at exactly three orthogonal vectors (see Figure 2(c) . The underlying clusters are detected correctly, except possibly for a few points at their intersection (see Figure 2(d)].

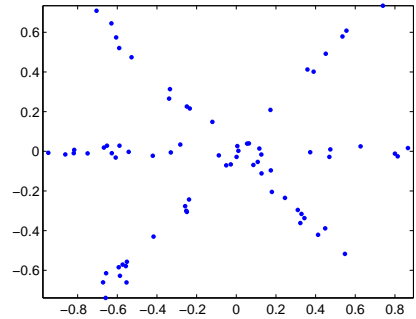

(a) data points

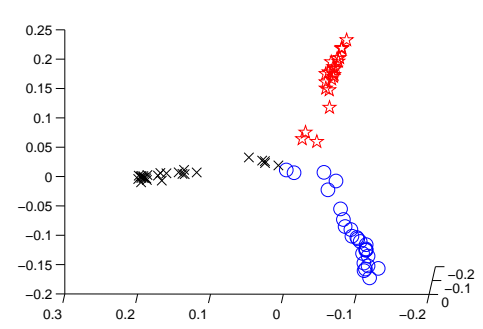

(c) rows of $\mathbf{U}$

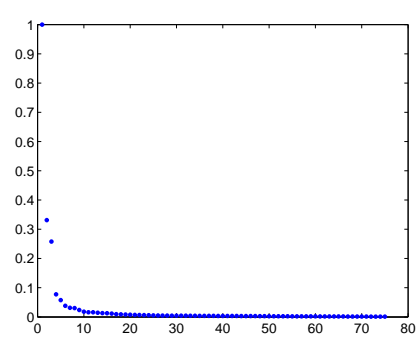

(b) eigenvalues of $\mathbf{Z}$

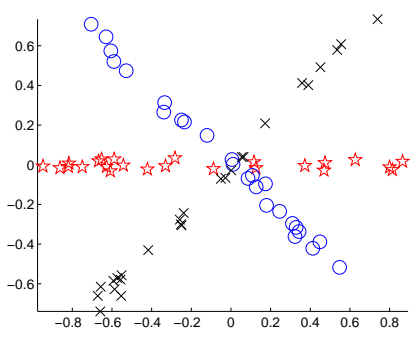

(d) detected clusters

Figure 2: Illustration of the perturbation analysis 

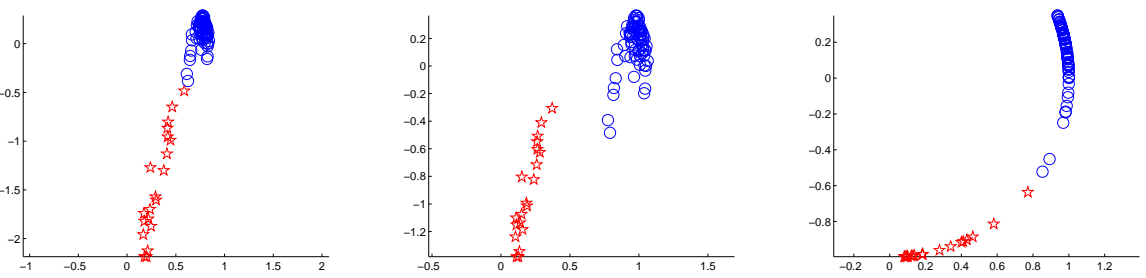

(a) The underlying clusters in the $\mathbf{U}, \mathbf{T}, \mathbf{V}$ spaces respectively
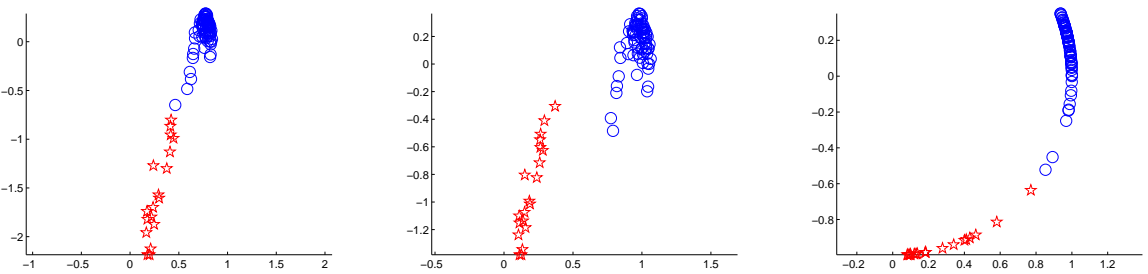

(b) The clusters found by $K$-means in the $\mathbf{U}, \mathbf{T}, \mathbf{V}$ spaces

Figure 3: The underlying clusters and those found by $K$-means in the $\mathbf{U}, \mathbf{T}, \mathbf{V}$ spaces. The given data consists of 80 and 20 points on two lines in $\mathbb{R}^{2}$. We note that, in order for the rows of $\mathbf{U}$ to have similar magnitudes to those of $\mathbf{T}$ and $\mathbf{V}$, we have scaled each row of $\mathbf{U}$ with the square root of the average cluster size, i.e., $\sqrt{N / K}$.

\subsection{The Effects of the Normalizations in TSCC}

\subsubsection{Possible Normalizations of $U$ and Their Effects on Clustering}

The analysis of the previous subsections uses the embedding represented by the rows of $\mathbf{U}$. It is possible to normalize these rows (e.g., by their lengths as in 31]) before applying $K$-means. In the following we consider two normalized versions of the rows of $\mathbf{U}$, and analyze their effects on the TSCC algorithm (in comparison with the rows of $\mathbf{U})$.

Using the cluster sizes, or the row lengths, one could normalize the matrix $\mathbf{U}$ and obtain two matrices $\mathbf{T}, \mathbf{V}$ whose rows are defined as follows:

$$
\begin{aligned}
\mathbf{t}^{(i)} & =\sqrt{N_{k}} \cdot \mathbf{u}^{(i)}, \quad i \in \mathrm{I}_{k}, 1 \leq k \leq K \\
\mathbf{v}^{(i)} & =\frac{1}{\left\|\mathbf{u}^{(i)}\right\|_{2}} \cdot \mathbf{u}^{(i)}, \quad 1 \leq i \leq N .
\end{aligned}
$$

These two normalizations are explained as follows. The $\mathbf{V}$ normalization discards all the magnitude information of the rows of $\mathbf{U}$ to contain only the angular information between them. The $\mathbf{T}$ normalization, containing the same angular information, reduces to $\mathbf{U}$ when $N_{1}=\cdots=N_{K}=N / K$, and otherwise tries to further separate the underlying clusters by scaling the rows using the cluster sizes. See Figure $3(\mathrm{a})$ for an illustration of the $\mathbf{U}, \mathbf{T}, \mathbf{V}$ spaces.

Remark 4.10. The normalization $\mathbf{T}$ assumes knowledge of the underlying cluster sizes, but can be effectively approximated without this knowledge when using our practical version of TSCC, i.e., SCC [7]. The SCC algorithm employs an 
iterative sampling procedure which converges quickly, thus it can estimate $\mathbf{T}$ in the current iteration by using the clusters obtained in the previous iteration.

We view the matrix $\mathbf{V}$ as a weak approximation to $\mathbf{T}$. Indeed, in the ideal case they coincide, since for all $1 \leq k \leq K$

$$
\left\|\widetilde{\mathbf{u}}^{(i)}\right\|_{2}=\frac{1}{\sqrt{N_{k}}}, \quad i \in \mathrm{I}_{k}
$$

(see equation (48) ). In the general case, the above equality only holds on average. More precisely, the orthonormality of $\mathbf{U}$ implies that

$$
\sum_{k=1}^{K} \sum_{i \in \mathrm{I}_{k}}\left\|\mathbf{u}^{(i)}\right\|_{2}^{2}=\|\mathbf{U}\|_{\mathrm{F}}^{2}=\sum_{j=1}^{K}\left\|\mathbf{u}_{j}\right\|_{2}^{2}=K
$$

We next define two criterions for analyzing the performance of $\mathbf{U}, \mathbf{T}$ and $\mathbf{V}$ when directly applying $K$-means to them.

First, we define a notion of the separation factor for the centers of the underlying clusters in each of the $\mathbf{U}, \mathbf{T}$ and $\mathbf{V}$ spaces. The separation factor of the centers in the $\mathbf{U}$ space is defined as follows:

$$
\beta(\mathbf{U}):=\frac{\sum_{1 \leq i<j \leq K}\left\langle\mathbf{c}^{(i)}, \mathbf{c}^{(j)}\right\rangle^{2}}{\left(\sum_{1 \leq k \leq K}\left\|\mathbf{c}^{(k)}\right\|_{2}^{2}\right)^{2}}
$$

The separation factors $\beta(\mathbf{T}), \beta(\mathbf{V})$ are defined similarly. The smaller $\beta$ is, the more separated in $\mathbb{R}^{K}$ the centers of the underlying clusters are. Lemma 4.2 directly implies that $\beta(\mathbf{T})$ is controlled by $\mathrm{TV}(\mathbf{U})$ as follows.

\section{Lemma 4.6.}

$$
\beta(\mathbf{T}) \leq \frac{\mathrm{TV}(\mathbf{U})}{(K-\mathrm{TV}(\mathbf{U}))^{2}} .
$$

We note that $\beta(\mathbf{U})=\beta(\mathbf{T})$ when $N_{k}=N / K, k=1, \ldots, K$. In general, we observe that $\beta(\mathbf{U}) \leq \beta(\mathbf{T}) \leq \beta(\mathbf{V})$, with the former two being fairly close. For example, $\beta(\mathbf{U})=.0004, \beta(\mathbf{T})=.0006, \beta(\mathbf{V})=.0032$ in Figure $3(\mathrm{a})$ In practice, however, we have found that the underlying clusters in the $\mathbf{U}, \mathbf{T}, \mathbf{V}$ spaces are usually not closely concentrated around their centers, thus this criterion may not be sufficient.

Second, we define a notion of the clustering identification error in the $\mathbf{U}, \mathbf{T}$ and $\mathbf{V}$ spaces respectively. For ease of discussion, we suppose that $K=2$. In the $\mathbf{U}$ space, the corresponding error has the form:

$$
e_{\mathrm{id}}(\mathbf{U}):=\frac{1}{N} \cdot \sum_{k=1}^{2} \#\left\{i \in \mathrm{I}_{k} \mid\left\|\mathbf{u}^{(i)}-\mathbf{c}^{(k)}\right\|_{2} \geq 1 / 2 \cdot\left\|\mathbf{c}^{(1)}-\mathbf{c}^{(2)}\right\|_{2}\right\}
$$

The errors in the $\mathbf{T}, \mathbf{V}$ spaces are defined similarly. The following lemma (proved in Appendix A.7) shows that both $e_{\mathrm{id}}(\mathbf{T})$ and $e_{\mathrm{id}}(\mathbf{U})$ can be controlled by $\mathrm{TV}(\mathbf{U})$, with the former having a smaller upper bound.

Lemma 4.7. Suppose that $K=2$. If

$$
\mathrm{TV}(\mathbf{U})<(\sqrt{3}-1)^{2}
$$


then the identification error in the $\mathbf{T}$ space is bounded above as follows:

$$
e_{\mathrm{id}}(\mathbf{T}) \leq \frac{4 \cdot \mathrm{TV}(\mathbf{U})}{2-\mathrm{TV}(\mathbf{U})-2 \sqrt{\mathrm{TV}(\mathbf{U})}}
$$

If

$$
\mathrm{TV}(\mathbf{U})<\left(\sqrt{2+\frac{4}{\varepsilon_{1}^{2}}}-\frac{2}{\varepsilon_{1}}\right)^{2},
$$

then the identification error in the $\mathbf{U}$ space is bounded above as follows:

$$
e_{\mathrm{id}}(\mathbf{U}) \leq \frac{4 \cdot \mathrm{TV}(\mathbf{U})}{2-\mathrm{TV}(\mathbf{U})-4 / \varepsilon_{1} \cdot \sqrt{\mathrm{TV}(\mathbf{U})}},
$$

where the constant $\varepsilon_{1}$ is defined in equation (12).

We remark that the clustering identification errors $e_{\mathrm{id}}(\mathbf{U}), e_{\mathrm{id}}(\mathbf{T}), e_{\mathrm{id}}(\mathbf{V})$ have only theoretical meanings. However, they can be used to estimate the clustering errors of $K$-means when applied in the $\mathbf{U}, \mathbf{T}, \mathbf{V}$ spaces respectively. We observed in practice that $e_{\mathrm{id}}(\mathbf{T})$ and $e_{\mathrm{id}}(\mathbf{V})$ are often very close.

Following the above discussion we think that $\mathbf{T}$ is probably the right normalization to be used in TSCC. Its practical implementation should follow Remark 4.10. We note that the application of this normalization in Lemma 4.2 results in analogous estimates for the $\mathbf{T}$ space which are independent of the sizes of clusters. Indeed, this normalization seems to outperform $\mathbf{U}$ when $N_{1}, \ldots, N_{K}$ vary widely (this claim is supported in practice by numerical experiments and in theory by Lemma 4.7). Another reason for our preference of $\mathbf{T}$ is that performing $K$-means in the $\mathbf{T}$ space is equivalent to performing weighted $K$-means (with weights $N_{k} / N, 1 \leq k \leq K$ ) in the $\mathbf{U}$ space, which allows small clusters to have relatively larger variance (see e.g., Figure 3(a)p.

The $\mathbf{V}$ normalization is another possibility to use in TSCC. On one hand, it is a weak approximation to $\mathbf{T}$; on the other hand, it contains only the angular information of the rows of $\mathbf{U}$. The use of only angular information for $K$-means clustering, partly supported by the polarization theorem in 6], seems to also separate the underlying clusters further. However, we need to understand this normalization more thoroughly, i.e., in terms of theoretical analysis.

In 7] we have used $\mathbf{U}$ to demonstrate our numerical strategies, which also apply to $\mathbf{T}$ and $\mathbf{V}$, and obtained good numerical results.

\subsubsection{TSCC Without Normalizing W}

We analyze here the TSCC algorithm when the matrix $\mathbf{W}$ is not normalized, i.e., skipping Step 3 of Algorithm 1 and letting $\mathbf{Z} \equiv \mathbf{W}$. We refer to the corresponding variant of TSCC as TSCC-UN, and formulate below analogous results of Proposition 4.1 and Theorem 4.5. The proof of Proposition4.8 directly follows that of Proposition 4.1 in Appendix A.1 (in particular, equations (42) and (43)). Theorem 4.9 is proved in Appendix A.6.

Proposition 4.8. Suppose that the TSCC-UN algorithm is applied with the perfect tensor $\widetilde{\mathbf{A}}$. Then 
1. The eigenvalues of $\widetilde{\mathbf{W}}$ are $\widetilde{d}_{K} \geq \cdots \geq \widetilde{d}_{2} \geq \widetilde{d}_{1}$ (each of multiplicity 1 ), and $\widetilde{\nu}_{K} \geq \cdots \geq \widetilde{\nu}_{2} \geq \widetilde{\nu}_{1}$ (of multiplicity $N_{K}, \ldots, N_{2}, N_{1}$ respectively), where

$$
\begin{aligned}
& \widetilde{d}_{k}:=\left(N_{k}-d-1\right) \cdot \mathrm{P}\left(N_{k}-1, d+1\right), \\
& \widetilde{\nu}_{k}:=(d+1) \cdot \mathrm{P}\left(N_{k}-2, d\right) .
\end{aligned}
$$

2. If $\widetilde{d}_{1}>\widetilde{\nu}_{K}$, the rows of $\widetilde{\mathbf{U}}$ are exactly $K$ mutually orthogonal vectors, each representing a distinct underlying cluster.

Theorem 4.9. Suppose that TSCC-UN is applied with a general affinity tensor $\mathcal{A}$, and that

$$
N \geq \sqrt{2(d+1)\left(1-\frac{K-1}{K} \varepsilon_{1}\right)^{d}\left(\frac{2 K}{\varepsilon_{1}}\right)^{d+2}},
$$

Let

$$
C_{2}\left(K, d, \varepsilon_{1}, \varepsilon_{2}\right):=32\left(\frac{2 K}{\varepsilon_{1}}\right)^{2(d+2)} .
$$

If

$$
N^{-(d+2)}\|\mathcal{E}\|_{\mathrm{F}}^{2} \leq \frac{1}{8 C_{2}}
$$

then

$$
\mathrm{TV}(\mathbf{U}) \leq C_{2} \cdot N^{-(d+2)}\|\mathcal{E}\|_{\mathrm{F}}^{2}
$$

In view of equation (28), the TSCC-UN algorithm seems to require large data size in order to work well. Numerical experiments also indicate that this approach is very sensitive to the variation of cluster sizes, and works consistently worse than the normalized approach, i.e., TSCC. Our current analysis, however, does not manifest the significant advantage of the normalized approach. We thus leave the related exploration to later research.

Von Luxburg et al. 40, have shown that in the framework of kernel spectral clustering, the normalized method is consistent under very general conditions. On the other hand, the unnormalized method is only consistent under very specific conditions that are rarely met in practice. Since $\mathbf{W}$ can be seen as a kernel matrix, 40. provides another evidence for our preference of the normalized approach.

\section{Probabilistic Analysis of TSCC}

In this section we analyze the performance of the TSCC algorithm with its own affinity tensor, i.e., the polar tensor of equation (6). We control with high probability (with respect to the sampling in Problem 1) the goodness of clustering of TSCC when applied to the data generated in Problem 1

\subsection{Basic Setting and Definitions}

We follow the setting of hybrid linear modeling described in Problem 1 together with the assumptions of regularity and possibly $d$-separation of $\left\{\mu_{i}\right\}_{i=1}^{K}$ (see 
Remark 2.5) as well as the restriction imposed by equation (12). We denote the corresponding $N$ random variables by $\mathfrak{X}_{1}, \ldots, \mathfrak{X}_{N} \in \mathbb{R}^{D}$ and maintain the previous notation for their sampled values $\mathbf{x}_{1}, \ldots, \mathbf{x}_{N}$. The joint sample space is $\left(\mathbb{R}^{D}\right)^{N}$, and the corresponding joint probability measure is

$$
\mu_{\mathrm{p}}:=\mu_{1}^{N_{1}} \times \cdots \times \mu_{K}^{N_{K}} .
$$

We introduce an incidence constant reflecting the separation between the measures $\mu_{1}, \ldots, \mu_{K}$ in regard to the polar curvature $c_{\mathrm{p}}$ and the tuning parameter $\sigma$. We first define the following sets

$$
\mathrm{S}_{k}:=\left(\operatorname{supp}\left(\mu_{k}\right)\right)^{d+2}, 1 \leq k \leq K .
$$

Then, given a constant $\sigma>0$, the incidence constant has the form:

$$
\begin{aligned}
& C_{\text {in }}\left(\mu_{1}, \ldots, \mu_{K} ; \sigma\right):= \\
& \max _{\substack{1 \leq k_{1}, \ldots, k_{d+2} \leq K \\
\text { not all equal }}} \int_{\mathrm{S}_{k_{1}}} \cdots \int_{\mathrm{S}_{k_{d+2}}} e^{\frac{-c_{\mathrm{p}}\left(\mathbf{z}_{1}, \ldots, \mathbf{z}_{d+2}\right)}{\sigma}} \mathrm{d} \mu_{k_{1}}\left(\mathbf{z}_{1}\right) \ldots \mathrm{d} \mu_{k_{d+2}}\left(\mathbf{z}_{d+2}\right),
\end{aligned}
$$

where the maximum is taken over all $1 \leq k_{1}, \ldots, k_{d+2} \leq K$ except $k_{1}=k_{2}=$ $\cdots=k_{d+2}$.

Remark 5.1. For TLSCC, the incidence constant is defined as follows:

$$
\begin{aligned}
& C_{\mathrm{in}, \mathrm{L}}\left(\mu_{1}, \ldots, \mu_{K} ; \sigma\right):= \\
& \max _{\substack{1 \leq k_{1}, \ldots, k_{d+1} \leq K \\
\text { not all equal }}} \int_{\mathrm{S}_{k_{1}}} \cdots \int_{\mathrm{S}_{k_{d+1}}} e^{\frac{-c_{\mathrm{p}}\left(\mathbf{0}, \mathbf{z}_{1}, \ldots, \mathbf{z}_{d+1}\right)}{\sigma}} \mathrm{d} \mu_{k_{1}}\left(\mathbf{z}_{1}\right) \ldots \mathrm{d} \mu_{k_{d+1}}\left(\mathbf{z}_{d+1}\right) .
\end{aligned}
$$

We note that for both TSCC and TLSCC, the incidence constant is between 0 and 1. The smaller the incidence constant is, the more separated (in terms of the polar curvature and the tuning parameter) the measures are. In Subsection 5.3 we estimate the incidence constant in a few special instances of hybrid linear modeling.

\subsection{The Main Result}

The following theorem (proved in Appendix A.9) shows that, when the underlying measures are sufficiently flat and well separated from each other, with high probability (with respect to the sampling of Problem 1) the TSCC algorithm segments the $K$ underlying clusters well.

Theorem 5.1. Suppose that the TSCC algorithm is applied to the data generated in Problem 1 with a tuning parameter $\sigma>0$. Let

$$
\alpha:=\frac{1}{\sigma^{2}} \sum_{k=1}^{K} c_{\mathrm{p}}^{2}\left(\mu_{k}\right)+C_{\mathrm{in}}\left(\mu_{1}, \ldots, \mu_{K} ; \sigma / 2\right),
$$

and $C_{1}=C_{1}\left(K, d, \varepsilon_{1}, \varepsilon_{2}\right)$ be the constant defined in Theorem 4.5. If

$$
\alpha<\frac{1}{16 C_{1}}
$$

then

$$
\mu_{\mathrm{p}}\left(\mathrm{TV}(\mathbf{U}) \leq 2 \alpha \cdot C_{1} \mid \text { Assumption 1 holds }\right) \geq 1-e^{-2 N \alpha^{2} /(d+2)^{2}} .
$$


Remark 5.2. Theorem 5.1 also holds for the TLSCC algorithm, but with $d$ replaced by $d-1$, and the constant $\alpha$ by

$$
\alpha_{\mathrm{L}}:=\frac{1}{\sigma^{2}} \sum_{k=1}^{K} c_{\mathrm{p}, \mathrm{L}}^{2}\left(\mu_{k}\right)+C_{\mathrm{in}, \mathrm{L}}\left(\mu_{1}, \ldots, \mu_{K} ; \sigma / 2\right),
$$

where for any Borel probability measure $\mu$,

$$
c_{\mathrm{p}, \mathrm{L}}(\mu):=\sqrt{\int c_{\mathrm{p}}^{2}\left(\mathbf{0}, \mathbf{z}_{1}, \ldots, \mathbf{z}_{d+1}\right) \mathrm{d} \mu\left(\mathbf{z}_{1}\right) \ldots \mathrm{d} \mu\left(\mathbf{z}_{d+1}\right)} .
$$

Remark 5.3. A similar version of Theorem 5.1 holds for general affinity tensors of the form $\left\{e^{-c\left(\mathbf{x}_{i_{1}}, \ldots, \mathbf{x}_{i_{d+2}}\right) / \sigma}\right\}_{1 \leq i_{1}, \ldots, i_{d+2} \leq N}$, where $c$ is a nonnegative, symmetric function defined on $\mathbb{R}^{d+2}$. The significance of using the polar curvature, or any other curvature satisfying Theorem 2.1, is explained in Subsection 5.3.

We showed in Lemma 4.7 that the clustering identification errors $e_{\mathrm{id}}(\mathbf{U})$ and $e_{\mathrm{id}}(\mathbf{T})$ can be controlled by $\mathrm{TV}(\mathbf{U})$ when $K=2$. Combining Lemma 4.7 and Theorem 5.1 yields the following probabilistic statement.

Corollary 5.2. Suppose that $K=2$, and that $\alpha, C_{1}$ are the constants defined in Theorem 5.1. If

$$
\alpha<\frac{1}{16 C_{1}}
$$

then

$$
\begin{aligned}
\mu_{\mathrm{p}}\left(e_{\mathrm{id}}(\mathbf{T})\right. & \left.\leq \frac{4 \alpha C_{1}}{1-\alpha C_{1}-\sqrt{2 \alpha C_{1}}} \mid \text { Assumption 1 holds }\right) \\
\geq & 1-e^{-2 N \alpha^{2} /(d+2)^{2}} .
\end{aligned}
$$

If

$$
\alpha<\frac{1}{2 C_{1}} \cdot \min \left(\frac{1}{8},\left(\sqrt{2+\frac{4}{\varepsilon_{1}^{2}}}-\frac{2}{\varepsilon_{1}}\right)^{2}\right),
$$

then

$$
\begin{aligned}
\mu_{\mathrm{p}}\left(e_{\mathrm{id}}(\mathbf{U})\right. & \left.\leq \frac{4 \alpha C_{1}}{1-\alpha C_{1}-2 / \varepsilon_{1} \cdot \sqrt{2 \alpha C_{1}}} \mid \text { Assumption [1 holds }\right) \\
\geq & 1-e^{-2 N \alpha^{2} /(d+2)^{2}} .
\end{aligned}
$$

\subsection{Interpretation of the Constant $\alpha$}

Theorem 5.1 shows the strong effect of the constant $\alpha$ on the goodness of clustering of the TSCC algorithm. This constant has two parts, which are explained respectively as follows.

Theorem 2.1 implies that the first part of $\alpha$ is comparable to

$$
\frac{1}{\sigma^{2}} \cdot \sum_{k=1}^{K} e_{2}^{2}\left(\mu_{k}\right)
$$

We thus view the first part as the sum of the within-cluster errors of the model scaled by $\sigma^{2}$. 
Remark 5.4. A similar interpretation applies to the tensors defined in equation (8). In this case, for any $q \geq 1$, the first term of $\alpha$ is replaced by

$$
\frac{1}{\sigma^{2}} \sum_{k=1}^{K} c_{\mathrm{p}}^{(2 q)}\left(\mu_{k}\right)
$$

where for any Borel probability measure $\mu$,

$$
c_{\mathrm{p}}^{(2 q)}(\mu):=\int c_{\mathrm{p}}^{2 q}\left(\mathbf{z}_{1}, \ldots, \mathbf{z}_{d+2}\right) \mathrm{d} \mu\left(\mathbf{z}_{1}\right) \ldots \mathrm{d} \mu\left(\mathbf{z}_{d+2}\right) .
$$

The above sum is then comparable to

$$
\frac{1}{\sigma^{2}} \cdot \sum_{k=1}^{K} e_{2 q}^{2 q}\left(\mu_{k}\right)
$$

where $e_{2 q}\left(\mu_{k}\right)$ is the error of approximating $\mu_{k}$ by a $d$-flat while minimizing the $\mathrm{L}_{2 q}$ norm [23].

We interpret the second part of $\alpha$, i.e., the incidence constant, as the betweenclusters interaction of the model. Unlike the first part, we do not have a theoretical result that fully establishes this interpretation. We show in a few special cases (with underlying linear subspaces) how to control this constant.

In the first example (Example 5.5) we estimate the incidence constant for two orthogonal line segments when using TSCC. The next three examples assume the use of the TLSCC algorithm. In Example 5.6 the model includes distributions along two clean line segments with an arbitrary angle $\theta$ between them. We establish the dependence of the incidence constant on $\theta$ and $\sigma$. In Example 5.7 we consider two orthogonal lines with uniform noise around them, and demonstrate the dependence of the incidence constant on the level of the noise and $\sigma$. Example 5.8 considers two clean orthogonal planes in $\mathbb{R}^{3}$.

Example 5.5. (TSCC: two orthogonal clean lines). We consider the following two orthogonal line segments in $\mathbb{R}^{2}$ :

$$
\text { L1 : } y=0, \quad 0 \leq x \leq L,
$$

and

$$
\text { L2: } x=0, \quad 0 \leq y \leq L,
$$

in which $L>0$ is a fixed constant. We assume arclength measures $\mu_{1}=\frac{\mathrm{d} x}{L}, \mu_{2}=$ $\frac{\mathrm{d} y}{L}$ supported on L1 and L2 respectively. For any $\sigma>0$, the incidence constant for TSCC is bounded as follows (see Appendix A.10):

$$
C_{\text {in }}\left(\mu_{1}, \mu_{2} ; \sigma\right) \leq \frac{\sigma}{\sqrt{2} L}\left(1-e^{-\sqrt{2} L / \sigma}\right)
$$

Example 5.6. (TLSCC: two intersecting clean lines). We consider the following two lines in $\mathbb{R}^{2}$ :

$$
\text { L1 : } y=0, \quad 0 \leq x \leq L,
$$

and

$$
\mathrm{L} 2: y=r \sin \theta, x=r \cos \theta, \quad 0 \leq r \leq L,
$$


in which $L>0$ and $0<\theta \leq \pi / 2$ are fixed constants. We assume arclength measures $\mu_{1}=\frac{\mathrm{d} x}{L}, \mu_{2}=\frac{\mathrm{d} r}{L}$ supported on L1 and L2 respectively. For any $\sigma>0$, the incidence constant for TLSCC is bounded as follows (see Appendix A.11):

$$
C_{\mathrm{in}, \mathrm{L}}\left(\mu_{1}, \mu_{2} ; \sigma\right) \leq 2\left(\frac{\sigma}{L \sin \theta}\right)^{2} \cdot\left(1-e^{-\frac{L \sin \theta}{\sigma}}\left(1+\frac{L \sin \theta}{\sigma}\right)\right)
$$

We note that when $\theta=\pi / 2, C_{\mathrm{in}, \mathrm{L}}$ has a faster decay rate than $C_{\text {in }}$ (see Example 5.5).

Example 5.7. (TLSCC: two orthogonal rectangles). We consider two rectangular strips in $\mathbb{R}^{2}$ determined by the following vertices respectively:

$$
\mathrm{R} 1:(\epsilon, 0),(L+\epsilon, 0),(\epsilon, \epsilon),(L+\epsilon, \epsilon),
$$

and

$$
\mathrm{R} 2:(0, \epsilon),(0, L+\epsilon),(\epsilon, \epsilon),(\epsilon, L+\epsilon),
$$

in which $0<\epsilon \ll L$. We assume uniform measures $\mu_{i}=\frac{1}{L \epsilon} \mathcal{L}_{2}$ restricted to $\mathrm{R} i$, $i=1,2$. We view $\mathrm{R} 1$ and $\mathrm{R} 2$ as two lines surrounded by uniform noise. Let $\omega:=L / \epsilon$. For any $\sigma>0$, the incidence constant for TLSCC has the following upper bound (see Appendix A.12)

$$
C_{\mathrm{in}, \mathrm{L}}\left(\mu_{1}, \mu_{2} ; \sigma\right) \leq \frac{\sqrt{\sigma}}{\omega^{2}}+\frac{2 \sqrt[4]{\sigma}}{\omega} \cdot e^{-1 /\left(2 \sigma^{3 / 4}\right)}+e^{-1 / \sigma^{3 / 4}} .
$$

In the limiting case of $\epsilon \rightarrow 0+$, i.e., when having two orthogonal lines with practically no noise, the above estimate decays faster to zero than the one in Example 5.6 with $\theta=\pi / 2$. This is due to the fact that in the current example we exclude the intersection of the two lines for any $\epsilon>0$. As it turned out, the limit of the corresponding integral (as $\epsilon \rightarrow 0+$ ) is not the same as the full integral of this limit.

Example 5.8. (TLSCC: two perpendicular clean half-disks). We consider the following portions of two unit disks (in polar coordinates) in $\mathbb{R}^{3}$ :

$$
\text { D1 : } x=0, y=\rho \cos \varphi, z=\rho \sin \varphi, \quad 0 \leq \rho \leq 1,0 \leq \varphi \leq \pi,
$$

and

$$
\text { D2 : } x=r \cos \theta, y=r \sin \theta, z=0, \quad 0 \leq r \leq 1,-\pi / 2 \leq \theta \leq \pi / 2 .
$$

We also assume uniform measures $\mu_{i}=\frac{2}{\pi} \mathcal{L}_{2}$ restricted on $\mathrm{D} i, i=1,2$. In this case, the incidence constant for TLSCC is bounded above by the following quantity (see Appendix A.13)

$$
C_{\mathrm{in}, \mathrm{L}}\left(\mu_{1}, \mu_{2} ; \sigma\right) \leq \frac{8 \sqrt{\sigma}}{\pi^{2}}+\frac{8 \sqrt[4]{\sigma}}{\pi}+\frac{4 \sigma^{2}}{(\sin \sqrt[4]{\sigma})^{4}}
$$

\subsection{On the Existence of Assumption 1}

The theory developed in this paper assumes that all affinity tensors used with TSCC, in particular the polar tensor, satisfy Assumption 1. We present some partial results regarding the existence of this assumption for the polar tensor 
while taking into account the restrictions on the size of $\sigma$ imposed by Theorem 5.1. We remark that those results also extend to some other tensors.

We first show in the following lemma (proved in Appendix A.8.1) that if a data is sampled from a hybrid linear model without noise, then Assumption 1 is always satisfied with the constant $\varepsilon_{2}=1$.

Lemma 5.3. If the TSCC is applied to data sampled from a mixture of clean d-flats, then

$$
\mathbf{D} \geq \widetilde{\mathbf{D}}
$$

For more general data sampled from a hybrid linear model with respect to a ball $B$ (according to Problem 1), one can easily obtain that Assumption 1 is satisfied with

$$
\varepsilon_{2}=e^{-\frac{2}{\sigma} \cdot \sqrt{d+2} \cdot \operatorname{diam}(B)}
$$

(see proof in Appendix A.8.2). However, since our main estimates depend inversely on $\varepsilon_{2}$ we would need to have the constant $\varepsilon_{2}$ sufficiently close to 1 , and thus the above equation implies a lower bound on $\sigma$ of the order of $\operatorname{diam}(B)$. On the other hand, the first term of the constant $\alpha$ stated in Theorem $5.1 \mathrm{im}$ plies an upper bound for $\sigma$ of the order of $\sum_{k=1}^{K} c_{\mathrm{p}}^{2}\left(\mu_{k}\right)$. These two bounds are rather contradictory (it is easy to see this in view of the interpretation of the $\operatorname{sum} \sum_{k=1}^{K} c_{\mathrm{p}}^{2}\left(\mu_{k}\right)$ in Subsection 5.3).

To resolve the above issue we can replace $\operatorname{diam}(B)$ in equation (40) with the term $\sum_{k=1}^{K} c_{\mathrm{p}}^{2}\left(\mu_{k}\right)$ and obtain the following estimate in expectation (see proof in Appendix A.8.3.

Lemma 5.4. If the TSCC is applied to data sampled according to Problem 1, then Assumption 1 holds in expectation in the following sense:

$$
E_{\mu_{\mathrm{p}}}(\mathbf{D}) \geq \varepsilon_{2} \cdot \widetilde{\mathbf{D}}
$$

where

$$
\varepsilon_{2}=e^{-\frac{2}{\sigma} \cdot \max _{1 \leq k \leq K} c_{\mathrm{p}}\left(\mu_{k}\right)} .
$$

Remark 5.9. We do not expect Assumption [1 to hold with high probability (i.e., having the measure $\mu_{\mathrm{p}}$ close to one) while maintaining the constant $\varepsilon_{2}$ formulated in Lemma 5.4. However, it seems reasonable to have a statement in high probability when replacing the polar curvature $c_{\mathrm{p}}\left(\mu_{k}\right)$ used in defining this constant with their following upper bounds:

$$
\widehat{c}_{\mathrm{p}}^{2}\left(\mu_{k}\right)=\max _{\mathbf{z}_{1} \in \operatorname{supp}\left(\mu_{k}\right)} \int c_{\mathrm{p}}^{2}\left(\mathbf{z}_{\mathbf{1}}, \mathbf{z}_{2}, \ldots, \mathbf{z}_{d+2}\right) \mathrm{d} \mu_{k}\left(\mathbf{z}_{2}\right) \ldots \mathrm{d} \mu_{k}\left(\mathbf{z}_{d+2}\right) .
$$

We leave the investigation of such a statement and the effect of using $\widehat{c}_{\mathrm{p}}^{2}(\mu)$ instead of $c_{\mathrm{p}}^{2}(\mu)$ to future research.

\section{Conclusion and Future Work}

We have analyzed the performance of TSCC in the setting of hybrid linear modeling. We first showed that we could precisely cluster the underlying components knowing the perfect tensor, and then established good performance in the case of reasonable deviation from the perfect case. Using this result, we 
proved that if a data set is sampled independently and identically according to the setting of Problem 11 then with high sampling probability the TSCC algorithm will perform well as long as the underlying distributions are sufficiently flat and separated. In [7] we develop a practical version of the TSCC algorithm by incorporating different numerical techniques and exemplify its successful performance using a number of artificial data sets and several real-world applications.

We conclude this paper by discussing both the open directions and the possible extensions of this work.

Further understanding of the two normalizations discussed in Subsection 4.3.1; We first explored in Subsection 4.3.1 possible normalizations of the matrix $\mathbf{U}$, and analyzed (to some extent) the performance of TSCC with and without them. We concluded that the normalization suggested by the matrix $\mathbf{T}$ is probably the right one to apply in TSCC. It will be interesting to test our practical strategy when applying such a normalization (see Remark 4.10) on both artificial and practical data sets with varying numbers of points within each cluster. Also, we wish to study more carefully the possible advantages of the normalization suggested by the matrix $\mathbf{V}$.

At last, Subsection 4.3.1 analyzed the TSCC algorithm when applied without the unnormalized matrix $\mathbf{Z}$. The perturbation results there were practically comparable to those obtained when applying TSCC with the normalized matrix $\mathbf{Z}$. It thus did not reveal the significant advantage of using $\mathbf{Z}$. In future investigations we would like to improve the current estimates so that they emphasize this significant advantage.

Further interpretation of the incidence constant: Currently we have described the behavior of the incidence constant in a few typical examples of two intersecting linear subspaces. We ask about characterization of this constant for general mixtures of flats, and its dependence on the separation between the subspaces, the magnitude of noise as well as the tuning parameter.

Estimation of the clustering identification error: We showed in Subsection 4.3.1 that when $K=2$ and $\mathrm{TV}(\mathbf{U})$ is sufficiently small, then a large percentage of the points can be clustered correctly. We would like to extend the corresponding analysis to the case where $K>2$.

Further investigation of Assumption 1: Assumption 1 is a crucial condition for Algorithm 1 to work well. Our partial results (i.e., Lemmas 5.3 and 5.4) showed that this assumption holds at least in expectation. We would like to explore the existence in high probability of Assumption 1 with a constant $\varepsilon_{2}>0$ that does not contradict the bounds imposed by Theorem 5.1 (see discussion in Section 5.4, in particular, Remark 5.9).

Analysis of other frameworks for multi-way clustering: Agarwal et al. 2] and Shashua et al. 32 suggested different frameworks for multi-way spectral clustering. It will be interesting to analyze the performance of their algorithms when applied to data sampled from a hybrid linear model.

Clustering flats in non-flat spaces, and even more general shapes: We are interested in generalizing the problem of clustering $d$-flats in Euclidean 
spaces to more general metric spaces where $d$-flats are replaced by $d$-dimensional geodesic surfaces. Also, we would like to modify our curvatures to cluster other shapes, e.g., circles, parabolas, spheres.

Detecting $d$-flats: We believe that it is possible to modify the methods described in this paper to detect an unknown $d$-flat in uniformly distributed background noise. If we are able to develop good curvatures for other geometric shapes, then we can generalize the detection problem to including such shapes (see [3] and references therein for other solutions to this problem).

\section{Acknowledgement}

We thank the reviewers and the action editor for valuable comments that have helped improve this paper; Ery Arias-Castro and Tyler Whitehouse for commenting on an earlier version of this manuscript; Ofer Zeitouni for clarifying to us Remark [5.9] Stefan Atev, Effrosyni Kokiopoulou, Yi Ma, Guillermo Sapiro, Arthur Szlam and Rene Vidal for valuable references; Dennis Cook, Peter Olver and Fadil Santosa for serving on the oral preliminary exam committee of GC, where the initial version of this work was discussed. We thank Mark Green, Kevin Vixie and IPAM for inviting us to participate in the 2005 Graduate Summer School on intelligent extraction of information from graphs and high dimensional data. GL also thanks Emmanuel Candes, Mark Green and IPAM for inviting him to participate in parts of the 2004 fall program on multiscale geometry and analysis in high dimensions. Both programs had a strong effect on this research. GL thanks Ingrid Daubechies who encouraged him to think about "hybrid linear modeling" already in 2002 (motivated by a brain imaging problem). The research presented in this paper is supported by NSF grant \#0612608.

\section{A Proofs}

\section{A.1 Proof of Proposition 4.1}

The affinity matrix $\widetilde{\mathbf{A}}$, the matricized version of $\widetilde{\mathcal{A}}$, is a $0 / 1$ matrix of size $N \times N^{d+1}$. We identify the unit entries in each row as follows. For any fixed $1 \leq i \leq N_{1}$, the entries of the $i^{\text {th }}$ row of $\widetilde{\mathbf{A}}$ are of the form $\widetilde{\mathcal{A}}\left(i, i_{2}, \ldots, i_{d+2}\right), 1 \leq$ $i_{2}, \ldots, i_{d+2} \leq N$. These entries will be 1 if they represent affinities of distinct $d+2$ points in $\widetilde{\mathrm{C}}_{1}$, that is, the indices $i, i_{2}, \ldots, i_{d+2}$ are distinct and between 1 and $N_{1}$. Therefore, the $i^{\text {th }}$ row has exactly $\mathrm{P}\left(N_{1}-1, d+1\right)$ entries filled by a 1 , which is exactly the number of permutations of size $d+1$ out of the first $N_{1}$ points excluding $i$. Similarly, each of the subsequent $N_{2}$ rows has $\mathrm{P}\left(N_{2}-1, d+1\right)$ ones, and each of the next $N_{3}$ rows has $\mathrm{P}\left(N_{3}-1, d+1\right)$ ones, etc..

The weight matrix $\widetilde{\mathbf{W}}=\widetilde{\mathbf{A}} \widetilde{\mathbf{A}}^{\prime}$ can be expressed in terms of the tensor $\widetilde{\mathcal{A}}$ in the following way:

$$
\widetilde{W}_{i j}=\sum_{1 \leq i_{2}, \ldots, i_{d+2} \leq N} \widetilde{\mathcal{A}}\left(i, i_{2}, \ldots, i_{d+2}\right) \widetilde{\mathcal{A}}\left(j, i_{2}, \ldots, i_{d+2}\right), \quad 1 \leq i, j \leq N .
$$


If $\mathbf{x}_{i}$ and $\mathbf{x}_{j}$ are not in the same underlying cluster, then all the products are zero. Therefore, $\widetilde{\mathbf{W}}$ is block diagonal:

$$
\widetilde{\mathbf{W}}=\operatorname{diag}\left\{\widetilde{\mathbf{W}}^{(1)}, \widetilde{\mathbf{W}}^{(2)}, \ldots, \widetilde{\mathbf{W}}^{(K)}\right\},
$$

where $\widetilde{\mathbf{W}}^{(k)} \in \mathbb{R}^{N_{k} \times N_{k}}$, corresponding to the underlying cluster $\widetilde{\mathrm{C}}_{k}$, has the following form:

$$
\widetilde{W}_{i j}^{(k)}= \begin{cases}\mathrm{P}\left(N_{k}-1, d+1\right), & \text { if } i=j ; \\ \mathrm{P}\left(N_{k}-2, d+1\right), & \text { otherwise. }\end{cases}
$$

Indeed, the diagonal elements of $\widetilde{\mathbf{W}}^{(k)}$ are simply the number of ones in the corresponding rows of $\widetilde{\mathbf{A}}$, and the off-diagonal elements are the number of ones that appear at the intersection of the corresponding pair of rows.

It then follows that

$$
\widetilde{\mathbf{D}}=\operatorname{diag}\{\widetilde{\mathbf{W}} \cdot \mathbf{1}\}=\operatorname{diag}\left\{\widetilde{d}_{1} \mathbf{I}_{N_{1}}, \widetilde{d}_{2} \mathbf{I}_{N_{2}}, \ldots, \widetilde{d}_{K} \mathbf{I}_{N_{K}}\right\},
$$

where

$$
\begin{aligned}
\widetilde{d}_{k} & =\mathrm{P}\left(N_{k}-1, d+1\right)+\left(N_{k}-1\right) \cdot \mathrm{P}\left(N_{k}-2, d+1\right) \\
& =\left(N_{k}-d-1\right) \cdot \mathrm{P}\left(N_{k}-1, d+1\right) .
\end{aligned}
$$

The normalized matrix $\widetilde{\mathbf{Z}}=\widetilde{\mathbf{D}}^{-1 / 2} \widetilde{\mathbf{W}} \widetilde{\mathbf{D}}^{-1 / 2}$ is also block diagonal:

$$
\widetilde{\mathbf{Z}}=\operatorname{diag}\left\{\widetilde{\mathbf{Z}}^{(1)}, \widetilde{\mathbf{Z}}^{(2)}, \ldots, \widetilde{\mathbf{Z}}^{(K)}\right\}
$$

where each block has the form $\widetilde{\mathbf{Z}}^{(k)}=\widetilde{\mathbf{W}}^{(k)} / \widetilde{d}_{k}, 1 \leq k \leq K$. The $(i, j)$-element of $\widetilde{\mathbf{Z}}^{(k)}$, for all $1 \leq i, j \leq N_{k}$, is

$$
\widetilde{Z}_{i j}^{(k)}= \begin{cases}\frac{1}{N_{k}-d-1}, & \text { if } i=j \\ \frac{N_{k}-d-2}{\left(N_{k}-1\right)\left(N_{k}-d-1\right)}, & \text { otherwise. }\end{cases}
$$

Straightforward calculation shows that each block matrix $\widetilde{\mathbf{Z}}^{(k)}$ has two distinct eigenvalues:

$$
\tilde{\lambda}_{n}^{(k)}= \begin{cases}1, & \text { if } n=1 \\ \frac{d+1}{\left(N_{k}-1\right)\left(N_{k}-d-1\right)}, & \text { if } 2 \leq n \leq N_{k}\end{cases}
$$

The eigenspace associated with the single eigenvalue 1 for $\widetilde{\mathbf{Z}}^{(k)}$ is spanned by $\mathbf{1}_{N_{k}}$, the $N_{k}$-dimensional column vector of all ones. Since the eigenvalues and eigenvectors of a block diagonal matrix are essentially the union of those of its blocks (for eigenvectors we need to append zeros in an appropriate way), we conclude that $\widetilde{\mathbf{Z}}$ has the largest eigenvalue 1 of multiplicity $K$ with associated eigenspace spanned by the following $K$ orthonormal vectors:

$$
\frac{1}{\sqrt{N_{1}}}\left(\begin{array}{c}
\mathbf{1}_{N_{1}} \\
\mathbf{0} \\
\vdots \\
\mathbf{0}
\end{array}\right), \frac{1}{\sqrt{N_{2}}}\left(\begin{array}{c}
\mathbf{0} \\
\mathbf{1}_{N_{2}} \\
\vdots \\
\mathbf{0}
\end{array}\right), \ldots, \frac{1}{\sqrt{N_{K}}}\left(\begin{array}{c}
\mathbf{0} \\
\vdots \\
\mathbf{0} \\
\mathbf{1}_{N_{K}}
\end{array}\right) \in \mathbb{R}^{N}
$$


We note that the $K$ eigenvectors associated with the eigenvalue 1 can only be determined up to an orthonormal transformation. That is,

$$
\widetilde{\mathbf{U}}=\left(\begin{array}{cccc}
\frac{1}{\sqrt{N_{1}}} \mathbf{1}_{N_{1}} & \mathbf{0} & \cdots & \mathbf{0} \\
\mathbf{0} & \frac{1}{\sqrt{N_{2}}} \mathbf{1}_{N_{2}} & \cdots & \mathbf{0} \\
\vdots & \vdots & \ddots & \vdots \\
\mathbf{0} & \mathbf{0} & \cdots & \frac{1}{\sqrt{N_{K}}} \mathbf{1}_{N_{K}}
\end{array}\right) \mathbf{Q} \in \mathbb{R}^{N \times K}
$$

where $\mathbf{Q}$ is a $K \times K$ orthonormal matrix.

If we write $\mathbf{Q}=\left(\mathbf{q}_{1}, \mathbf{q}_{2}, \ldots, \mathbf{q}_{K}\right)^{\prime}$, where $\mathbf{q}_{k}$ is the $k^{\text {th }}$ column of $\mathbf{Q}^{\prime}$, then equation (48) implies that the $K$ clusters are mapped one-to-one to the $K$ mutually orthogonal vectors $\frac{1}{\sqrt{N_{1}}} \cdot \mathbf{q}_{1}, \ldots, \frac{1}{\sqrt{N_{K}}} \cdot \mathbf{q}_{K} \in \mathbb{R}^{K}$.

\section{A.2 Proof of Lemma 4.4}

We first note that $P^{K}(\mathbf{Z})=\mathbf{U U}^{\prime}$ and $P^{K}(\widetilde{\mathbf{Z}})=\widetilde{\mathbf{U}} \widetilde{\mathbf{U}}^{\prime}$, due to the fact that both $\mathbf{U}$ and $\widetilde{\mathbf{U}}$ are composed of orthonormal columns. Therefore,

$$
\begin{aligned}
\left\|P^{K}(\mathbf{Z})-P^{K}(\widetilde{\mathbf{Z}})\right\|_{\mathrm{F}}^{2} & =\left\|\mathbf{U} \mathbf{U}^{\prime}-\widetilde{\mathbf{U}} \widetilde{\mathbf{U}}^{\prime}\right\|_{\mathrm{F}}^{2}=\operatorname{trace}\left(\left(\mathbf{U} \mathbf{U}^{\prime}-\widetilde{\mathbf{U}^{\prime}}\right)^{2}\right) \\
& =\operatorname{trace}\left(\mathbf{U} \mathbf{U}^{\prime}-\mathbf{U} \mathbf{U}^{\prime} \tilde{\mathbf{U}} \widetilde{\mathbf{U}^{\prime}}-\widetilde{\mathbf{U}} \widetilde{\mathbf{U}}^{\prime} \mathbf{U} \mathbf{U}^{\prime}+\widetilde{\mathbf{U}^{\prime}}\right) .
\end{aligned}
$$

Since

$$
\operatorname{trace}\left(\mathbf{U U}^{\prime}\right)=\operatorname{trace}\left(\mathbf{U}^{\prime} \mathbf{U}\right)=\operatorname{trace}\left(\mathbf{I}_{K}\right)=K \text {, }
$$

and similarly,

$$
\operatorname{trace}\left(\widetilde{\mathbf{U}} \widetilde{\mathbf{U}}^{\prime}\right)=K
$$

we have

$$
\left\|P^{K}(\mathbf{Z})-P^{K}(\widetilde{\mathbf{Z}})\right\|_{\mathrm{F}}^{2}=2 K-2 \cdot \operatorname{trace}\left(\mathbf{U U}^{\prime} \widetilde{\mathbf{U}^{\prime}}\right)
$$

In the formula of the matrix $\widetilde{\mathbf{U}}$ (equation (48)), there is an arbitrary orthonormal matrix $\mathbf{Q}$. However, the product $\widetilde{\mathbf{U}} \widetilde{\mathbf{U}}^{\prime}$ does not depend on $\mathbf{Q}$. Hence, we can use a representation of $\widetilde{\mathbf{U}}$ where $\mathbf{Q}$ is the identity matrix, and proceed as follows:

$$
\begin{aligned}
\left\|P^{K}(\mathbf{Z})-P^{K}(\widetilde{\mathbf{Z}})\right\|_{\mathrm{F}}^{2} & =2 K-2 \cdot\left\|\mathbf{U}^{\prime} \widetilde{\mathbf{U}}\right\|_{\mathrm{F}}^{2} \\
& =2 K-2 \cdot\left\|\left[\sum_{i \in \mathrm{I}_{1}} \frac{1}{\sqrt{N_{1}}}\left(\mathbf{u}^{(i)}\right)^{\prime} \cdots \sum_{i \in \mathrm{I}_{K}} \frac{1}{\sqrt{N_{K}}}\left(\mathbf{u}^{(i)}\right)^{\prime}\right]\right\|_{\mathrm{F}}^{2} \\
& =2 K-2 \cdot \sum_{k=1}^{K} \frac{1}{N_{k}}\left\|\sum_{i \in \mathrm{I}_{k}} \mathbf{u}^{(i)}\right\|_{2}^{2} \\
& =2 K-2 \cdot \sum_{k=1}^{K} N_{k}\left\|\mathbf{c}^{(k)}\right\|_{2}^{2} \cdot
\end{aligned}
$$


Since the columns of the matrix $\mathbf{U}$ are unit vectors, we have

$$
\sum_{i=1}^{N}\left\|\mathbf{u}^{(i)}\right\|_{2}^{2}=\|\mathbf{U}\|_{\mathrm{F}}^{2}=\sum_{k=1}^{K}\left\|\mathbf{u}_{k}\right\|_{2}^{2}=K .
$$

Combining the last two equations we get that

$$
\begin{aligned}
\left\|P^{K}(\mathbf{Z})-P^{K}(\widetilde{\mathbf{Z}})\right\|_{\mathrm{F}}^{2} & =2 \cdot\left(\sum_{i=1}^{N}\left\|\mathbf{u}^{(i)}\right\|_{2}^{2}-\sum_{k=1}^{K} N_{k} \cdot\left\|\mathbf{c}^{(k)}\right\|_{2}^{2}\right) \\
& =2 \cdot \sum_{k=1}^{K}\left(\sum_{i \in \mathrm{I}_{k}}\left\|\mathbf{u}^{(i)}\right\|_{2}^{2}-N_{k} \cdot\left\|\mathbf{c}^{(k)}\right\|_{2}^{2}\right) \\
& =2 \cdot \sum_{k=1}^{K} \sum_{i \in \mathrm{I}_{k}}\left\|\mathbf{u}^{(i)}-\mathbf{c}^{(k)}\right\|_{2}^{2} .
\end{aligned}
$$

\section{A.3 Proof of Lemma 4.2}

Equation (14) is a direct consequence of combining equation (49) and Lemma 4.4

To show equation (15), we first expand the following two products

$$
\begin{aligned}
\mathbf{U U}^{\prime} & =\left(\left\langle\mathbf{u}^{(i)}, \mathbf{u}^{(j)}\right\rangle\right)_{1 \leq i, j \leq N}, \\
\widetilde{\mathbf{U}} \widetilde{\mathbf{U}}^{\prime} & =\operatorname{diag}\left\{\frac{1}{N_{1}} \mathbf{1}_{N_{1} \times N_{1}}, \ldots, \frac{1}{N_{K}} \mathbf{1}_{N_{K} \times N_{K}}\right\} .
\end{aligned}
$$

Then

$$
\begin{aligned}
& \left\|P^{K}(\mathbf{Z})-P^{K}(\widetilde{\mathbf{Z}})\right\|_{\mathrm{F}}^{2}=\left\|\mathbf{U} \mathbf{U}^{\prime}-\widetilde{\mathbf{U}} \widetilde{\mathbf{U}}^{\prime}\right\|_{\mathrm{F}}^{2} \\
& =\sum_{1 \leq k \leq K} \sum_{i, j \in \mathrm{I}_{k}}\left(\left\langle\mathbf{u}^{(i)}, \mathbf{u}^{(j)}\right\rangle-\frac{1}{N_{k}}\right)^{2}+\sum_{1 \leq k \neq \ell \leq K} \sum_{i \in \mathrm{I}_{k}, j \in I_{\ell}}\left(\left\langle\mathbf{u}^{(i)}, \mathbf{u}^{(j)}\right\rangle\right)^{2} \\
& \geq \sum_{1 \leq k \neq \ell \leq K} \sum_{i \in \mathrm{I}_{k}, j \in I_{\ell}}\left(\left\langle\mathbf{u}^{(i)}, \mathbf{u}^{(j)}\right\rangle\right)^{2}
\end{aligned}
$$

We next apply the inequality $\left(\sum_{i=1}^{m} a_{i}\right)^{2} \leq m \cdot \sum_{i=1}^{m} a_{i}^{2}$ and conclude that

$$
\begin{aligned}
\left\|P^{K}(\mathbf{Z})-P^{K}(\widetilde{\mathbf{Z}})\right\|_{\mathrm{F}}^{2} & \geq \sum_{1 \leq k \neq \ell \leq K} \frac{1}{N_{k} N_{\ell}} \cdot\left(\sum_{i \in I_{k}, j \in I_{\ell}}\left\langle\mathbf{u}^{(i)}, \mathbf{u}^{(j)}\right\rangle\right)^{2} \\
& =\sum_{1 \leq k \neq \ell \leq K} N_{k} N_{\ell} \cdot\left\langle\mathbf{c}^{(k)}, \mathbf{c}^{(\ell)}\right\rangle^{2}
\end{aligned}
$$

Finally, combining the last equation and Lemma 4.4 completes the proof. 


\section{A.4 Review of Principal Angles and Proof of Lemma 4.3}

\section{Review of Principal Angles}

The principal angles $0 \leq \theta_{1} \leq \cdots \leq \theta_{K} \leq \pi / 2$ between two $K$-dimensional subspaces $S$ and $T$ are defined recursively as follows (see e.g., [10]):

$$
\begin{aligned}
\cos \theta_{1} & =\max _{\mathbf{x} \in S,\|\mathbf{x}\|_{2}=1} \max _{\mathbf{y} \in T,\|\mathbf{y}\|_{2}=1} \mathbf{x}^{\prime} \mathbf{y}=\mathbf{x}_{1}^{\prime} \mathbf{y}_{1}, \\
\cos \theta_{2}= & \max _{\substack{\mathbf{x} \in S,\|\mathbf{x}\|_{2}=1 \\
\mathbf{x} \perp \mathbf{x}_{1}}} \max _{\mathbf{y} \in T,\|\mathbf{y}\|_{2}=1}^{\mathbf{y} \perp \mathbf{y}_{1}} \mathbf{x}^{\prime} \mathbf{y}=\mathbf{x}_{2}^{\prime} \mathbf{y}_{2}, \\
\ldots & \max _{\cos \theta_{K}=} \max _{\substack{\mathbf{x} \in S,\|\mathbf{x}\|_{2}=1 \\
\mathbf{x} \perp\left\{\mathbf{x}_{1}, \ldots, \mathbf{x}_{K-1}\right\}}} \mathbf{x}_{\substack{\mathbf{y} \in T,\|\mathbf{y}\|_{2}=1 \\
\mathbf{y} \perp\left\{\mathbf{y}_{1}, \ldots, \mathbf{y}_{K-1}\right\}}} \mathbf{x}^{\prime} \mathbf{y}=\mathbf{x}_{K}^{\prime} \mathbf{y}_{K} .
\end{aligned}
$$

Another formula for the cosines of the principal angles is obtained as follows. Let $\mathbf{S}$ and $\mathbf{T}$ be two matrices whose columns define orthonormal bases of $S$ and $T$ respectively. Since any $\mathbf{x} \in S$ and $\mathbf{y} \in T$ can be represented as $\mathbf{x}=\mathbf{S u}$ and $\mathbf{y}=\mathbf{T} \mathbf{v}$ respectively, where $\mathbf{u}$ and $\mathbf{v}$ are unit vectors in $\mathbb{R}^{K}$, it follows that

$$
\cos \theta_{k}=\sigma_{k}\left(\mathbf{S}^{\prime} \mathbf{T}\right) \text { for } 1 \leq k \leq K,
$$

where $\sigma_{k}\left(\mathbf{S}^{\prime} \mathbf{T}\right)$ denotes the $k^{\text {th }}$ largest singular value of $\mathbf{S}^{\prime} \mathbf{T}$.

\section{Proof of Lemma 4.3}

From the proof of Lemma 4.4 we have that

$$
\begin{aligned}
\left\|P^{K}(\mathbf{Z})-P^{K}(\widetilde{\mathbf{Z}})\right\|_{\mathrm{F}}^{2} & =2 K-2\left\|\mathbf{U}^{\prime} \widetilde{\mathbf{U}}\right\|_{\mathrm{F}}^{2}=2 K-2 \sum_{k=1}^{K} \sigma_{k}^{2}\left(\mathbf{U}^{\prime} \widetilde{\mathbf{U}}\right) \\
& =2 K-2 \sum_{k=1}^{K} \cos ^{2} \theta_{k}=2 \sum_{k=1}^{K} \sin ^{2} \theta_{k} .
\end{aligned}
$$

\section{A.5 Proof of Theorem 4.5}

The proof is based on a perturbation result by Zwald and Blanchard 43, Theorem 3]. In fact, we only need a special case of it which is formulated below.

Theorem A.1 (Matrix version of Theorem 3 in Zwald and Blanchard [4]). Let $\mathbf{S}$ be a symmetric positive square matrix with nonzero eigenvalues $\lambda_{1} \geq \cdots \geq$ $\lambda_{K}>\lambda_{K+1} \geq \cdots \geq 0$, where $K>0$ is an integer. Define $\delta_{K}=\lambda_{K}-\lambda_{K+1}>0$, which denotes the $K^{\text {th }}$ eigengap of $\mathbf{S}$. Let $\mathbf{B}$ be another symmetric matrix such that $\|\mathbf{B}\|_{\mathrm{F}}<\delta_{K} / 4$ and $\mathbf{S}+\mathbf{B}$ is still a positive matrix. Then

$$
\left\|P^{K}(\mathbf{S}+\mathbf{B})-P^{K}(\mathbf{S})\right\|_{\mathrm{F}} \leq 2\|\mathbf{B}\|_{\mathrm{F}} / \delta_{K}
$$

In order to apply the above theorem to the quantity $\left\|P^{K}(\mathbf{Z})-P^{K}(\widetilde{\mathbf{Z}})\right\|_{\mathrm{F}}$, we need a lower bound on $\widetilde{\delta}_{K}$, the $K^{\text {th }}$ eigengap of $\widetilde{\mathbf{Z}}$, and an upper bound on the Frobenius norm of the difference $\mathbf{B}:=\mathbf{Z}-\widetilde{\mathbf{Z}}$. While the former bound is immediate, we find the latter bound somewhat challenging. 
First, equation (47), together with $N_{1}=\min _{1 \leq k \leq K} N_{k}$, implies that:

$$
\widetilde{\delta}_{K}=1-\frac{d+1}{\left(N_{1}-1\right)\left(N_{1}-d-1\right)} .
$$

Since $N_{1} \geq 2(d+1)+1$ by equation (12), we then obtain that

$$
\widetilde{\delta}_{K} \geq \frac{2 d+3}{2 d+4} \geq \frac{3}{4} .
$$

Next, we estimate the Frobenius norm of the perturbation $\mathbf{B}$ as follows. Using the definitions of the matrices $\mathbf{Z}$ and $\mathbf{W}$, we rewrite $\mathbf{B}$ in the following way:

$$
\mathbf{B}=\mathbf{D}^{-1 / 2} \mathbf{A} \mathbf{A}^{\prime} \mathbf{D}^{-1 / 2}-\widetilde{\mathbf{D}}^{-1 / 2} \widetilde{\mathbf{A}} \widetilde{\mathbf{A}}^{\prime} \widetilde{\mathbf{D}}^{-1 / 2}
$$

Regrouping terms gives that

$$
\begin{aligned}
\mathbf{B}= & \left(\mathbf{D}^{-1 / 2} \mathbf{A}-\widetilde{\mathbf{D}}^{-1 / 2} \widetilde{\mathbf{A}}\right)\left(\mathbf{D}^{-1 / 2} \mathbf{A}-\widetilde{\mathbf{D}}^{-1 / 2} \widetilde{\mathbf{A}}\right)^{\prime} \\
& +\left(\mathbf{D}^{-1 / 2} \mathbf{A}-\widetilde{\mathbf{D}}^{-1 / 2} \widetilde{\mathbf{A}}\right) \widetilde{\mathbf{A}}^{\prime} \widetilde{\mathbf{D}}^{-1 / 2}+\widetilde{\mathbf{D}}^{-1 / 2} \widetilde{\mathbf{A}}\left(\mathbf{D}^{-1 / 2} \mathbf{A}-\widetilde{\mathbf{D}}^{-1 / 2} \widetilde{\mathbf{A}}\right)^{\prime}
\end{aligned}
$$

We thus get an initial upper bound on its Frobenius norm:

$$
\|\mathbf{B}\|_{\mathrm{F}} \leq\left\|\mathbf{D}^{-1 / 2} \mathbf{A}-\widetilde{\mathbf{D}}^{-1 / 2} \widetilde{\mathbf{A}}\right\|_{\mathrm{F}}^{2}+2\left\|\widetilde{\mathbf{D}}^{-1 / 2} \widetilde{\mathbf{A}}\right\|_{\mathrm{F}}\left\|\mathbf{D}^{-1 / 2} \mathbf{A}-\widetilde{\mathbf{D}}^{-1 / 2} \widetilde{\mathbf{A}}\right\|_{\mathrm{F}} .
$$

By using equations (45) and (46), we get that

$$
\left\|\widetilde{\mathbf{D}}^{-1 / 2} \widetilde{\mathbf{A}}\right\|_{\mathrm{F}}^{2}=\operatorname{trace}\left(\widetilde{\mathbf{D}}^{-1 / 2} \widetilde{\mathbf{W}} \widetilde{\mathbf{D}}^{-1 / 2}\right)=\operatorname{trace}(\widetilde{\mathbf{Z}})=\sum_{k=1}^{K} \frac{N_{k}}{N_{k}-d-1} .
$$

Equation (12) implies that

$$
\frac{N_{k}}{N_{k}-d-1}<2, \quad 1 \leq k \leq K .
$$

Consequently, we have

$$
\left\|\widetilde{\mathbf{D}}^{-1 / 2} \widetilde{\mathbf{A}}\right\|_{\mathrm{F}}^{2}<2 K
$$

and thus equation (58) becomes

$$
\|\mathbf{B}\|_{\mathrm{F}}<\left\|\mathbf{D}^{-1 / 2} \mathbf{A}-\widetilde{\mathbf{D}}^{-1 / 2} \widetilde{\mathbf{A}}\right\|_{\mathrm{F}}^{2}+2 \sqrt{2 K} \cdot\left\|\mathbf{D}^{-1 / 2} \mathbf{A}-\widetilde{\mathbf{D}}^{-1 / 2} \widetilde{\mathbf{A}}\right\|_{\mathrm{F}} .
$$

Therefore, in order to control $\|\mathbf{B}\|_{\mathrm{F}}$, we only need to bound $\left\|\mathbf{D}^{-1 / 2} \mathbf{A}-\widetilde{\mathbf{D}}^{-1 / 2} \widetilde{\mathbf{A}}\right\|_{\mathrm{F}}$.

Let

$$
\mathbf{E}:=\mathbf{A}-\widetilde{\mathbf{A}} .
$$

Replacing $\mathbf{A}$ with $\widetilde{\mathbf{A}}+\mathbf{E}$ yields that

$$
\begin{aligned}
\left\|\mathbf{D}^{-1 / 2} \mathbf{A}-\widetilde{\mathbf{D}}^{-1 / 2} \widetilde{\mathbf{A}}\right\|_{\mathrm{F}} & =\left\|\left(\mathbf{D}^{-1 / 2}-\widetilde{\mathbf{D}}^{-1 / 2}\right) \widetilde{\mathbf{A}}+\mathbf{D}^{-1 / 2} \mathbf{E}\right\|_{\mathrm{F}} \\
& \leq\left\|\left(\mathbf{D}^{-1 / 2}-\widetilde{\mathbf{D}}^{-1 / 2}\right) \widetilde{\mathbf{A}}\right\|_{\mathrm{F}}+\left\|\mathbf{D}^{-1 / 2} \mathbf{E}\right\|_{\mathrm{F}}
\end{aligned}
$$


The second term on the right hand side of equation (60) is bounded as follows

$$
\begin{aligned}
\left\|\mathbf{D}^{-1 / 2} \mathbf{E}\right\|_{\mathrm{F}} & \leq\left\|\mathbf{D}^{-1 / 2}\right\|_{2} \cdot\|\mathbf{E}\|_{\mathrm{F}} \leq\left\|\left(\varepsilon_{2} \widetilde{\mathbf{D}}\right)^{-1 / 2}\right\|_{2} \cdot\|\mathbf{E}\|_{\mathrm{F}} \\
& =\left(\varepsilon_{2} \widetilde{d}_{1}\right)^{-1 / 2} \cdot\|\mathbf{E}\|_{\mathrm{F}}
\end{aligned}
$$

in which the second inequality follows from Assumption 1 ( $\left.\mathbf{D} \geq \varepsilon_{2} \widetilde{\mathbf{D}}>0\right)$, and the last equality is due to our convention: $N_{1}=\min _{1 \leq k \leq K} N_{k}$ (which implies that $\widetilde{d}_{1}=\min _{1 \leq k \leq K} \widetilde{d}_{k}$ ).

Bounding the first term of the right hand side of equation (60) requires more work. We estimate it as follows:

$$
\begin{aligned}
\left\|\left(\mathbf{D}^{-1 / 2}-\widetilde{\mathbf{D}}^{-1 / 2}\right) \cdot \widetilde{\mathbf{A}}\right\|_{\mathrm{F}} & =\left\|\widetilde{\mathbf{D}}^{-1 / 2} \mathbf{D}^{-1 / 2}\left(\mathbf{D}^{1 / 2}+\widetilde{\mathbf{D}}^{1 / 2}\right)^{-1}(\mathbf{D}-\widetilde{\mathbf{D}}) \cdot \widetilde{\mathbf{A}}\right\|_{\mathrm{F}} \\
& \leq\left\|\widetilde{\mathbf{D}}^{-1 / 2}\left(\varepsilon_{2} \widetilde{\mathbf{D}}\right)^{-1 / 2}\left(\widetilde{\mathbf{D}}^{1 / 2}\right)^{-1}(\mathbf{D}-\widetilde{\mathbf{D}}) \cdot \widetilde{\mathbf{A}}\right\|_{\mathrm{F}} \\
& =\varepsilon_{2}^{-1 / 2}\left\|\widetilde{\mathbf{D}}^{-3 / 2}(\mathbf{D}-\widetilde{\mathbf{D}}) \cdot \widetilde{\mathbf{A}}\right\|_{\mathrm{F}}
\end{aligned}
$$

We proceed by using the index sets $I_{1}, \ldots, I_{K}$ (see equation (9)) to expand the last equation:

$$
\begin{aligned}
\left\|\left(\mathbf{D}^{-1 / 2}-\widetilde{\mathbf{D}}^{-1 / 2}\right) \cdot \widetilde{\mathbf{A}}\right\|_{\mathrm{F}} & \leq \varepsilon_{2}^{-1 / 2} \sqrt{\sum_{1 \leq k \leq K} \sum_{i \in \mathrm{I}_{k}}\left(D_{i i}-\widetilde{d}_{k}\right)^{2} \widetilde{d}_{k}^{-3} \cdot\|\widetilde{\mathbf{A}}(i,:)\|_{2}^{2}} \\
& =\varepsilon_{2}^{-1 / 2} \sqrt{\sum_{1 \leq k \leq K} \sum_{i \in \mathrm{I}_{k}} \frac{\left(D_{i i}-\widetilde{d}_{k}\right)^{2}}{\left(N_{k}-d-1\right) \cdot \widetilde{d}_{k}^{2}}} \\
& \leq \varepsilon_{2}^{-1 / 2} \widetilde{d}_{1}^{-1}\left(N_{1}-d-1\right)^{-1 / 2} \cdot\|\mathbf{D}-\widetilde{\mathbf{D}}\|_{\mathrm{F}} \cdot
\end{aligned}
$$

Using the definitions of $\mathbf{D}$ and $\widetilde{\mathbf{D}}$, we obtain that

$$
\begin{aligned}
\|\mathbf{D}-\widetilde{\mathbf{D}}\|_{\mathrm{F}} & =\left\|(\mathbf{W}-\widetilde{\mathbf{W}}) \cdot \mathbf{1}_{N}\right\|_{2} \leq\|\mathbf{W}-\widetilde{\mathbf{W}}\|_{\mathrm{F}} \cdot\left\|\mathbf{1}_{N}\right\|_{2} \\
& =N^{1 / 2} \cdot\left\|\widetilde{\mathbf{A}} \mathbf{E}^{\prime}+\mathbf{E} \widetilde{\mathbf{A}}^{\prime}+\mathbf{E} \mathbf{E}^{\prime}\right\|_{\mathrm{F}} \\
& \leq N^{1 / 2} \cdot\left(2\|\widetilde{\mathbf{A}}\|_{\mathrm{F}}\|\mathbf{E}\|_{\mathrm{F}}+\|\mathbf{E}\|_{\mathrm{F}}^{2}\right) .
\end{aligned}
$$

Combining equations (63) and (64) and applying $N_{1}-d-1>\frac{N_{1}}{2} \geq \frac{\varepsilon_{1} N}{2 K}$ (following equation (12)) gives that

$$
\left\|\left(\mathbf{D}^{-1 / 2}-\widetilde{\mathbf{D}}^{-1 / 2}\right) \widetilde{\mathbf{A}}\right\|_{\mathrm{F}} \leq\left(\frac{2 K}{\varepsilon_{1} \varepsilon_{2}}\right)^{1 / 2} \tilde{d}_{1}^{-1}\left(2\|\widetilde{\mathbf{A}}\|_{\mathrm{F}}\|\mathbf{E}\|_{\mathrm{F}}+\|\mathbf{E}\|_{\mathrm{F}}^{2}\right) .
$$

By substituting equations (61) and (65) into equation (60), we arrive at

$$
\begin{aligned}
\left\|\mathbf{D}^{-1 / 2} \mathbf{A}-\widetilde{\mathbf{D}}^{-1 / 2} \widetilde{\mathbf{A}}\right\|_{\mathrm{F}} \leq & \left(\frac{2 K}{\varepsilon_{1} \varepsilon_{2}}\right)^{1 / 2} \widetilde{d}_{1}^{-1}\left(2\|\widetilde{\mathbf{A}}\|_{\mathrm{F}}\|\mathbf{E}\|_{\mathrm{F}}+\|\mathbf{E}\|_{\mathrm{F}}^{2}\right) \\
& +\varepsilon_{2}^{-1 / 2} \widetilde{d}_{1}^{-1 / 2}\|\mathbf{E}\|_{\mathrm{F}} .
\end{aligned}
$$


In order to complete the above estimate for $\left\|\mathbf{D}^{-1 / 2} \mathbf{A}-\widetilde{\mathbf{D}}^{-1 / 2} \widetilde{\mathbf{A}}\right\|_{\mathrm{F}}$, we need to estimate $\|\widetilde{\mathbf{A}}\|_{F}$ from above

$$
\|\widetilde{\mathbf{A}}\|_{\mathrm{F}}<\sqrt{N^{d+2}}=N^{(d+2) / 2}
$$

and $\widetilde{d}_{1}$ from below

$$
\widetilde{d}_{1}=\left(N_{1}-d-1\right) \cdot \mathrm{P}\left(N_{1}-1, d+1\right) \geq\left(N_{1} / 2\right)^{d+2} \geq\left(\frac{\varepsilon_{1} N}{2 K}\right)^{d+2}
$$

We also note that all the elements of the matrix $\mathbf{E}$ are between -1 and 1 , and thus

$$
\|\mathbf{E}\|_{\mathrm{F}} \leq N^{(d+2) / 2} .
$$

We then continue from equation (66), together with the last three estimates, and get that

$$
\begin{aligned}
& \left\|\mathbf{D}^{-1 / 2} \mathbf{A}-\widetilde{\mathbf{D}}^{-1 / 2} \widetilde{\mathbf{A}}\right\|_{\mathrm{F}} \\
& \leq\left(\frac{2 K}{\varepsilon_{1} \varepsilon_{2}}\right)^{1 / 2}\left(\frac{\varepsilon_{1} N}{2 K}\right)^{-(d+2)} 3 N^{(d+2) / 2}\|\mathbf{E}\|_{\mathrm{F}}+\varepsilon_{2}^{-1 / 2}\left(\frac{\varepsilon_{1} N}{2 K}\right)^{-(d+2) / 2}\|\mathbf{E}\|_{\mathrm{F}} \\
& \leq 4 \varepsilon_{2}^{-1 / 2}\left(\frac{2 K}{\varepsilon_{1}}\right)^{d+5 / 2} N^{-(d+2) / 2}\|\mathbf{E}\|_{\mathrm{F}}
\end{aligned}
$$

Finally, it follows from equations (59) and (67) that

$$
\|\mathbf{B}\|_{\mathrm{F}} \leq C_{0}\left(K, d, \varepsilon_{1}, \varepsilon_{2}\right) \cdot N^{-(d+2) / 2}\|\mathbf{E}\|_{\mathrm{F}},
$$

where

$$
C_{0}\left(K, d, \varepsilon_{1}, \varepsilon_{2}\right):=16 \varepsilon_{2}^{-1}\left(\frac{2 K}{\varepsilon_{1}}\right)^{2 d+5}+2 \sqrt{2 K} \cdot 4 \varepsilon_{2}^{-1 / 2}\left(\frac{2 K}{\varepsilon_{1}}\right)^{d+5 / 2} .
$$

By combining Theorem A.1 with equations (57) and (68), we obtain that when

$$
C_{0}\left(K, d, \varepsilon_{1}, \varepsilon_{2}\right) \cdot N^{-(d+2) / 2}\|\mathbf{E}\|_{\mathrm{F}}<3 / 16
$$

then

$$
\left\|P^{K}(\mathbf{Z})-P^{K}(\widetilde{\mathbf{Z}})\right\|_{\mathrm{F}} \leq 8 / 3 \cdot C_{0}\left(K, d, \varepsilon_{1}, \varepsilon_{2}\right) \cdot N^{-(d+2) / 2}\|\mathbf{E}\|_{\mathrm{F}}
$$

Letting

$$
C_{1}\left(K, d, \varepsilon_{1}, \varepsilon_{2}\right):=32 / 9 \cdot C_{0}^{2}\left(K, d, \varepsilon_{1}, \varepsilon_{2}\right)
$$

and noting

$$
\|\mathbf{E}\|_{\mathrm{F}} \equiv\|\mathcal{E}\|_{\mathrm{F}},
$$

we complete the proof by combining Lemma 4.4 and equations (70) and (171). 


\section{A.6 Proof of Theorem 4.9}

The proof proceeds in parallel to that of Theorem 4.5. That is, we bound from below the $K^{\text {th }}$ eigengap $\widetilde{\delta}_{K}$ of $\widetilde{\mathbf{W}}$, estimate from above the Frobenius norm of the perturbation $\mathbf{B}:=\mathbf{W}-\widetilde{\mathbf{W}}$, and then conclude the theorem by combining these two bounds with Theorem A.1.

Straightforward calculation shows that the matrix $\widetilde{\mathbf{W}}$ (see formula in Equation (43)) has the following eigenvalues:

$$
\widetilde{d}_{K} \geq \cdots \geq \widetilde{d}_{2} \geq \widetilde{d}_{1} \text { and } \nu_{K} \geq \cdots \geq \nu_{2} \geq \nu_{1},
$$

where $\widetilde{d}_{k}, 1 \leq k \leq K$, are defined in equation (26), and

$$
\nu_{k}:=(d+1) \cdot \mathrm{P}\left(N_{k}-2, d\right), \quad k=1, \ldots, K .
$$

Using equation (12) we obtain that

$$
N_{K}=N-\sum_{k=1}^{K-1} N_{k} \leq N-(K-1) \cdot \frac{\varepsilon_{1} N}{K}=\left(1-\frac{K-1}{K} \varepsilon_{1}\right) \cdot N .
$$

The above equation together with equations (12) and (28) implies that

$$
\begin{aligned}
\widetilde{\delta}_{K} & =\widetilde{d}_{1}-\nu_{K} \\
& \geq\left(\frac{N_{1}}{2}\right)^{d+2}-(d+1) \cdot N_{K}{ }^{d} \\
& \geq\left(\frac{\varepsilon_{1} N}{2 K}\right)^{d+2}-(d+1) \cdot\left(1-\frac{K-1}{K} \varepsilon_{1}\right)^{d} N^{d} \\
& \geq \frac{1}{2}\left(\frac{\varepsilon_{1} N}{2 K}\right)^{d+2} .
\end{aligned}
$$

We follow by bounding the magnitude of the perturbation $\mathbf{B}=\widetilde{\mathbf{W}}-\mathbf{W}$ :

$$
\|\mathbf{B}\|_{\mathrm{F}}=\left\|\mathbf{A} \mathbf{E}^{\prime}+\mathbf{E} \widetilde{\mathbf{A}}^{\prime}\right\|_{\mathrm{F}} \leq\|\mathbf{A}\|_{\mathrm{F}}\|\mathbf{E}\|_{\mathrm{F}}+\|\mathbf{E}\|_{\mathrm{F}}\|\widetilde{\mathbf{A}}\|_{\mathrm{F}} \leq 2 N^{(d+2) / 2}\|\mathbf{E}\|_{\mathrm{F}} .
$$

Therefore, by combining equations (73) and (74) with Theorem A.1 we conclude that when

$$
N^{-(d+2) / 2}\|\mathbf{E}\|_{\mathrm{F}} \leq \frac{1}{16}\left(\frac{\varepsilon_{1}}{2 K}\right)^{d+2},
$$

we have

$$
\left\|P^{K}(\mathbf{W})-P^{K}(\widetilde{\mathbf{W}})\right\|_{\mathrm{F}} \leq 8\left(\frac{2 K}{\varepsilon_{1}}\right)^{d+2} N^{-(d+2) / 2}\|\mathbf{E}\|_{\mathrm{F}} .
$$

Theorem 4.9 is then a direct consequence of combining the above equation and Lemma 4.4 .

\section{A.7 Proof of Lemma 4.7}

In the $\mathbf{T}$ space the centers of the underlying clusters are

$$
\mathbf{c}_{\mathbf{T}}^{(k)}:=\sqrt{N_{k}} \cdot \mathbf{c}^{(k)}, \quad 1 \leq k \leq K .
$$


Applying Lemma 4.2 with $K=2$ gives that

$$
\begin{aligned}
\left\|\mathbf{c}_{\mathbf{T}}^{(1)}-\mathbf{c}_{\mathbf{T}}^{(2)}\right\|_{2}^{2} & =N_{1} \cdot\left\|\mathbf{c}^{(1)}\right\|_{2}^{2}+N_{2} \cdot\left\|\mathbf{c}^{(2)}\right\|_{2}^{2}-2 \sqrt{N_{1} N_{2}} \cdot\left\langle\mathbf{c}^{(1)}, \mathbf{c}^{(2)}\right\rangle \\
& \geq 2-\operatorname{TV}(\mathbf{U})-2 \sqrt{\operatorname{TV}(\mathbf{U})} .
\end{aligned}
$$

When

$$
\operatorname{TV}(\mathbf{U})<(\sqrt{3}-1)^{2}
$$

we can let

$$
\tau:=\sqrt{2-\mathrm{TV}(\mathbf{U})-2 \sqrt{\mathrm{TV}(\mathbf{U})}}
$$

Then the clustering identification error of TSCC in the $\mathbf{T}$ space is bounded as follows:

$$
e_{\mathrm{id}}(\mathbf{T}) \leq \frac{1}{N} \cdot \sum_{k=1}^{2} \#\left\{i \in \mathrm{I}_{k} \mid\left\|\mathbf{t}^{(i)}-\mathbf{c}_{\mathbf{T}}^{(k)}\right\|_{2} \geq \tau / 2\right\} .
$$

For each $k=1,2$, we apply Chebyshev's inequality and obtain that

$$
\#\left\{i \in \mathrm{I}_{k} \mid\left\|\mathbf{t}^{(i)}-\mathbf{c}_{\mathbf{T}}^{(k)}\right\|_{2} \geq \tau / 2\right\} \leq \frac{4}{\tau^{2}} \sum_{i \in \mathrm{I}_{k}}\left\|\mathbf{t}^{(i)}-\mathbf{c}_{\mathbf{T}}^{(k)}\right\|_{2}^{2} .
$$

Thus,

$$
\begin{aligned}
e_{\mathrm{id}}(\mathbf{T}) & \leq \frac{1}{N} \cdot \sum_{k=1}^{2} \frac{4}{\tau^{2}} \sum_{i \in \mathrm{I}_{k}}\left\|\mathbf{t}^{(i)}-\mathbf{c}_{\mathbf{T}}^{(k)}\right\|_{2}^{2} \\
& \leq \frac{4}{\tau^{2}} \sum_{k=1}^{2} \frac{N_{k}}{N} \sum_{i \in \mathrm{I}_{k}}\left\|\mathbf{u}^{(i)}-\mathbf{c}^{(k)}\right\|_{2}^{2} \\
& \leq \frac{4}{\tau^{2}} \cdot \operatorname{TV}(\mathbf{U}) .
\end{aligned}
$$

In the $\mathbf{U}$ space, we also apply Lemma 4.2 with $K=2$, together with the assumptions $N_{2} \geq N_{1} \geq \varepsilon_{1} \cdot N / 2$, and obtain that

$$
\begin{aligned}
\left\|\mathbf{c}^{(1)}-\mathbf{c}^{(2)}\right\|_{2}^{2} & =\left\|\mathbf{c}^{(1)}\right\|_{2}^{2}+\left\|\mathbf{c}^{(2)}\right\|_{2}^{2}-2 \cdot\left\langle\mathbf{c}^{(1)}, \mathbf{c}^{(2)}\right\rangle \\
& \geq \frac{1}{N_{2}} \cdot\left(N_{1}\left\|\mathbf{c}^{(1)}\right\|_{2}^{2}+N_{2}\left\|\mathbf{c}^{(2)}\right\|_{2}^{2}\right)-\frac{2}{\sqrt{N_{1} N_{2}}} \cdot \sqrt{N_{1} N_{2}}\left\langle\mathbf{c}^{(1)}, \mathbf{c}^{(2)}\right\rangle \\
& \geq \frac{1}{N_{2}} \cdot(2-\mathrm{TV}(\mathbf{U}))-\frac{2}{N_{1}} \sqrt{\mathrm{TV}(\mathbf{U})} \\
& \geq \frac{1}{N} \cdot\left(2-\mathrm{TV}(\mathbf{U})-4 / \varepsilon_{1} \cdot \sqrt{\mathrm{TV}(\mathbf{U})}\right)
\end{aligned}
$$

When

$$
\mathrm{TV}(\mathbf{U})<\left(\sqrt{2+\frac{4}{\varepsilon_{1}^{2}}}-\frac{2}{\varepsilon_{1}}\right)^{2}
$$

we can apply similar steps as above to obtain that

$$
e_{\mathrm{id}}(\mathbf{U}) \leq \frac{4 \mathrm{TV}(\mathbf{U})}{2-\mathrm{TV}(\mathbf{U})-4 / \varepsilon_{1} \cdot \sqrt{\mathrm{TV}(\mathbf{U})}}
$$




\section{A.8 Proofs of Main Statements of Subsection 5.4}

\section{A.8.1 Proof of Lemma 5.3}

For any $1 \leq k \leq K$ and $i \in \mathrm{I}_{k}$, we have

$$
\begin{aligned}
& D_{i i} \geq \sum_{j \in \mathrm{I}_{k}} W_{i j} \geq \sum_{j \in \mathrm{I}_{k}} \sum_{i_{2}, \ldots, i_{d+2} \in \mathrm{I}_{k}} \mathcal{A}\left(i, i_{2}, \ldots, i_{d+2}\right) \mathcal{A}\left(j, i_{2}, \ldots, i_{d+2}\right)
\end{aligned}
$$

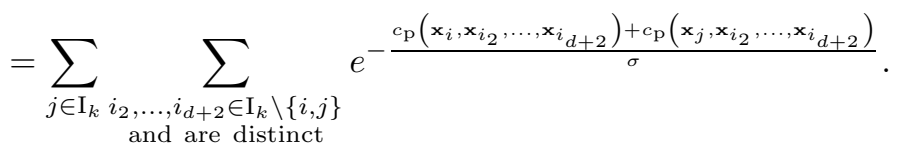

When the given data is noiseless, the polar curvature of any distinct $d+2$ points in $\widetilde{\mathrm{C}}_{k}$ is zero. Hence,

$$
D_{i i} \geq \sum_{j \in \mathrm{I}_{k}} \sum_{\substack{i_{2}, \ldots, i_{d+2} \in \mathrm{I}_{k} \backslash\{i, j\} \\ \text { and are distinct }}} 1=\widetilde{d}_{k},
$$

where $\widetilde{d}_{k}$ (replicated $N_{k}$ times), $1 \leq k \leq K$, are the diagonal elements of $\widetilde{\mathbf{D}}$ (see equation (26) $)$. We have thus proved that $\mathbf{D} \geq \widetilde{\mathbf{D}}$.

\section{A.8.2 Proof of Equation (40)}

We note the following obvious bound on the polar curvature of any sampled points $\mathbf{x}_{i_{1}}, \ldots, \mathbf{x}_{i_{d+2}}$ from the ball $B$ according to Problem 1 .

$$
c_{\mathrm{p}}\left(\mathbf{x}_{i_{1}}, \mathbf{x}_{i_{2}}, \ldots, \mathbf{x}_{i_{d+2}}\right) \leq \operatorname{diam}(B) \cdot \sqrt{d+2} .
$$

Combining this bound with equation (76) we obtain that

$$
D_{i i} \geq \sum_{j \in \mathrm{I}_{k}} \sum_{\substack{i_{2}, \ldots, i_{d+2} \in \mathrm{I}_{k} \backslash\{i, j\} \\ \text { and are distinct }}} e^{-\frac{\sqrt{d+2} \operatorname{diam}(B)}{\sigma}} e^{-\frac{\sqrt{d+2} \operatorname{diam}(B)}{\sigma}}=e^{-\frac{2 \sqrt{d+2} \operatorname{diam}(B)}{\sigma}} \cdot \widetilde{d}_{k} .
$$

\section{A.8.3 Proof of Lemma 5.4}

We take the expectation of each side of equation (76) with respect to the measure $\mu_{\mathrm{p}}$ (defined in equation (30) ), and proceed using Jensen's inequality (twice) as follows:

$$
\begin{aligned}
E_{\mu_{\mathrm{p}}}\left(D_{i i}\right) & \geq \sum_{j \in \mathrm{I}_{k}} \sum_{\substack{i_{2}, \ldots, i_{d+2} \in \mathrm{I}_{k} \backslash\{i, j\} \\
\text { and are distinct }}} e^{-\frac{2}{\sigma} \cdot E_{\mu_{k}}^{d+2} c_{\mathrm{p}}\left(\mathfrak{X}_{i}, \mathfrak{X}_{i_{2}}, \ldots, \mathfrak{X}_{i_{d+2}}\right)} \\
& \geq \sum_{j \in \mathrm{I}_{k}} \sum_{\substack{i_{2}, \ldots, i_{d+2} \in \mathrm{I}_{k} \backslash\{i, j\} \\
\text { and are distinct }}} e^{-\frac{2}{\sigma} \cdot \sqrt{E_{\mu_{k} d+2} c_{\mathrm{p}}^{2}\left(\mathfrak{X}_{i}, \mathfrak{X}_{i_{2}}, \ldots, \mathfrak{X}_{i_{d+2}}\right)}} \\
& =e^{-\frac{2}{\sigma} \cdot c_{\mathrm{p}}\left(\mu_{k}\right)} \cdot \widetilde{d}_{k},
\end{aligned}
$$

where in the last step we have used equation (44). Letting

$$
\varepsilon_{2}:=\min _{1 \leq k \leq K} e^{-\frac{2}{\sigma} \cdot c_{\mathrm{p}}\left(\mu_{k}\right)}=e^{-\frac{2}{\sigma} \cdot \max _{1 \leq k \leq K} c_{\mathrm{p}}\left(\mu_{k}\right)},
$$


we have that

$$
E_{\mu_{\mathrm{p}}}\left(D_{i i}\right) \geq \varepsilon_{2} \cdot \widetilde{d}_{k}, \quad i \in \mathrm{I}_{k}, 1 \leq k \leq K
$$

Equivalently,

$$
E_{\mu_{\mathrm{p}}}(\mathbf{D}) \geq \varepsilon_{2} \cdot \widetilde{\mathbf{D}}
$$

\section{A.9 Proof of Theorem 5.1}

We first bound the expectation of the perturbation $\left\|\mathcal{E}_{\mathrm{p}}\right\|_{\mathrm{F}}^{2}$, where $\mathcal{E}_{\mathrm{p}}=\mathcal{A}_{\mathrm{p}}-\widetilde{\mathcal{A}}$, and then apply McDiarmid's inequality [29] to obtain a probabilistic estimate for $\left\|\mathcal{E}_{\mathrm{p}}\right\|_{\mathrm{F}}^{2}$. Finally, we conclude the proof by combining the probabilistic estimate together with Theorem 4.5 .

Using the definitions of the sets $\mathrm{I}_{1}, \ldots, \mathrm{I}_{K}$ and the tensors $\mathcal{A}_{\mathrm{p}}$ and $\widetilde{\mathcal{A}}$, we express $\left\|\mathcal{E}_{\mathrm{p}}\right\|_{\mathrm{F}}^{2}$ as a function of the random variables $\mathfrak{X}_{1}, \ldots, \mathfrak{X}_{N}$ :

$$
\left\|\mathcal{E}_{\mathrm{p}}\right\|_{\mathrm{F}}^{2}=\sum_{k=1}^{K} \sum_{\mathrm{I}_{k}^{d+2}}\left(1-e^{\frac{-c_{\mathrm{p}}\left(\mathfrak{x}_{i_{1}}, \ldots, \mathfrak{x}_{i}{ }_{d+2}\right)}{\sigma}}\right)^{2}+\sum_{\left(\bigcup_{k=1}^{K} \mathrm{I}_{k}^{d+2}\right)^{c}}\left(e^{\left.\frac{-c_{\mathrm{p}}\left(\mathfrak{x}_{i_{1}}, \ldots, \mathfrak{x}_{i}{ }\right)}{\sigma}\right)}\right)^{2} .
$$

By applying the inequality: $1-e^{-|x|} \leq|x|$, we obtain that

$$
\left\|\mathcal{E}_{\mathrm{p}}\right\|_{\mathrm{F}}^{2} \leq \sum_{k=1}^{K} \sum_{\mathrm{I}_{k}^{d+2}} \frac{c_{\mathrm{p}}^{2}\left(\mathfrak{X}_{i_{1}}, \ldots, \mathfrak{X}_{i_{d+2}}\right)}{\sigma^{2}}+\sum_{\left(\bigcup_{k=1}^{K} \mathrm{I}_{k}^{d+2}\right)^{c}} e^{\frac{-c_{\mathrm{p}}\left(\mathfrak{X}_{i_{1}}, \ldots, \mathfrak{X}_{i_{d+2}}\right)}{\sigma / 2}} .
$$

We then take the expectation of $\left\|\mathcal{E}_{\mathrm{p}}\right\|_{\mathrm{F}}^{2}$ (with respect to $\mu_{\mathrm{p}}$ ) using equations (4) and (31) and have that

$$
\begin{aligned}
E_{\mu_{\mathrm{p}}}\left(\left\|\mathcal{E}_{\mathrm{p}}\right\|_{\mathrm{F}}^{2}\right) & \leq \frac{1}{\sigma^{2}} \sum_{k=1}^{K} N_{k}^{d+2} c_{\mathrm{p}}^{2}\left(\mu_{k}\right)+N^{d+2} C_{\mathrm{in}}\left(\mu_{1}, \ldots, \mu_{K} ; \sigma / 2\right) \\
& =N^{d+2} \cdot\left(\frac{1}{\sigma^{2}} \sum_{k=1}^{K}\left(\frac{N_{k}}{N}\right)^{d+2} c_{\mathrm{p}}^{2}\left(\mu_{k}\right)+C_{\mathrm{in}}\left(\mu_{1}, \ldots, \mu_{K} ; \sigma / 2\right)\right) \\
& \leq \alpha \cdot N^{d+2}
\end{aligned}
$$

in which

$$
\alpha:=\frac{1}{\sigma^{2}} \cdot \sum_{k=1}^{K} c_{\mathrm{p}}^{2}\left(\mu_{k}\right)+C_{\mathrm{in}}\left(\mu_{1}, \ldots, \mu_{K} ; \sigma / 2\right) .
$$

We next note that for each fixed $1 \leq i \leq N$,

$\sup _{\mathfrak{X}_{1}, \ldots, \mathfrak{X}_{N}, \widehat{\mathfrak{X}}_{i}}\left|\left\|\mathcal{E}_{\mathrm{p}}\right\|_{\mathrm{F}}^{2}\left(\mathfrak{X}_{1}, \ldots, \mathfrak{X}_{i}, \ldots, \mathfrak{X}_{N}\right)-\left\|\mathcal{E}_{\mathrm{p}}\right\|_{\mathrm{F}}^{2}\left(\mathfrak{X}_{1}, \ldots, \widehat{\mathfrak{X}}_{i}, \ldots, \mathfrak{X}_{N}\right)\right| \leq(d+2) \cdot N^{d+1}$.

Indeed, the number of additive terms in $\left\|\mathcal{E}_{\mathrm{p}}\right\|_{\mathrm{F}}^{2}\left(\mathfrak{X}_{1}, \ldots, \mathfrak{X}_{N}\right)$ that contain $\mathfrak{X}_{i}$ is $(d+2) \cdot \mathrm{P}(N-1, d+1)$, and each of them is between 0 and 1 .

The above property implies that $\left\|\mathcal{E}_{\mathrm{p}}\right\|_{\mathrm{F}}^{2}$ satisfies McDiarmid's inequality [29], that is,

$$
\mu_{\mathrm{p}}\left(\left\|\mathcal{E}_{\mathrm{p}}\right\|_{\mathrm{F}}^{2}-E_{\mu_{\mathrm{p}}}\left(\left\|\mathcal{E}_{\mathrm{p}}\right\|_{\mathrm{F}}^{2}\right) \geq \alpha N^{d+2}\right) \leq e^{-2 N \alpha^{2} /(d+2)^{2}}
$$


Combining the last equation with equation (77) yields that

$$
\mu_{\mathrm{p}}\left(\|\mathcal{E}\|_{\mathrm{F}}^{2} \geq 2 \alpha N^{d+2}\right) \leq e^{-2 N \alpha^{2} /(d+2)^{2}},
$$

or equivalently,

$$
\mu_{\mathrm{p}}\left(N^{-(d+2)}\left\|\mathcal{E}_{\mathrm{p}}\right\|_{\mathrm{F}}^{2}<2 \alpha\right) \geq 1-e^{-2 N \alpha^{2} /(d+2)^{2}} .
$$

Consequently, combining Theorem 4.5 and the last equation gives that, if

$$
2 \alpha \leq \frac{1}{8 C_{1}} \text {, where } C_{1}=C_{1}\left(K, d, \varepsilon_{1}, \varepsilon_{2}\right) \text { is defined in equation (71), }
$$

then

$$
\begin{aligned}
& \mu_{\mathrm{p}}\left(\mathrm{TV}(\mathbf{U})<2 \alpha \cdot C_{1} \mid \text { Assumption 1 holds }\right) \\
& \geq \mu_{\mathrm{p}}\left(\mathrm{TV}(\mathbf{U})<2 \alpha \cdot C_{1} \mid \text { Assumption 1 holds, and } N^{-(d+2)}\left\|\mathcal{E}_{\mathrm{p}}\right\|_{\mathrm{F}}^{2}<2 \alpha\right) \\
& \quad \cdot \mu_{\mathrm{p}}\left(N^{-(d+2)}\left\|\mathcal{E}_{\mathrm{p}}\right\|_{\mathrm{F}}^{2}<2 \alpha \mid \text { Assumption 1 holds }\right) \\
& =1 \cdot \mu_{\mathrm{p}}\left(N^{-(d+2)}\left\|\mathcal{E}_{\mathrm{p}}\right\|_{\mathrm{F}}^{2}<2 \alpha\right) \\
& \geq 1-e^{-2 N \alpha^{2} /(d+2)^{2}} .
\end{aligned}
$$

\section{A.10 Proof of Equation (36) in Example 5.5}

For any three points $\mathbf{p}_{1}\left(x_{1}, 0\right), \mathbf{p}_{2}\left(x_{2}, 0\right) \in \mathrm{L} 1$, and $\mathbf{q}(0, y) \in \mathrm{L} 2$, their polar curvature is bounded below by

$$
\begin{aligned}
c_{\mathrm{p}}\left(\mathbf{p}_{1}, \mathbf{p}_{2}, \mathbf{q}\right) & =\operatorname{diam}\left\{\mathbf{p}_{1}, \mathbf{p}_{2}, \mathbf{q}\right\} \cdot \sqrt{\sin ^{2} \angle \mathbf{p}_{1} \mathbf{p}_{2} \mathbf{q}+\sin ^{2} \angle \mathbf{p}_{2} \mathbf{p}_{1} \mathbf{q}+\sin ^{2} \angle \mathbf{p}_{1} \mathbf{q} \mathbf{p}_{2}} \\
& \geq \max \left(\sqrt{x_{1}^{2}+y^{2}}, \sqrt{x_{2}^{2}+y^{2}}\right) \cdot \sqrt{\frac{y^{2}}{x_{1}^{2}+y^{2}}+\frac{y^{2}}{x_{2}^{2}+y^{2}}} \\
& \geq \sqrt{y^{2}+y^{2}}=\sqrt{2} \cdot y .
\end{aligned}
$$

Thus, by using the symmetry of the lines, we obtain that

$$
\begin{aligned}
C_{\mathrm{in}}\left(\mu_{1}, \mu_{2} ; \sigma\right) & =\int_{\mathrm{L} 1} \int_{\mathrm{L} 1} \int_{\mathrm{L} 2} e^{-\frac{c_{\mathrm{p}}\left(\mathbf{p}_{1}, \mathbf{p}_{2}, \mathbf{q}\right)}{\sigma}} \mathrm{d} \mu_{1}\left(\mathbf{p}_{1}\right) \mathrm{d} \mu_{1}\left(\mathbf{p}_{2}\right) \mathrm{d} \mu_{2}(\mathbf{q}) \\
& \leq \int_{0}^{L} e^{-\frac{\sqrt{2} y}{\sigma}} \frac{\mathrm{d} y}{L}=\frac{\sigma}{\sqrt{2} L}\left(1-e^{-\sqrt{2} L / \sigma}\right) .
\end{aligned}
$$

\section{A.11 Proof of Equation (37) in Example 5.6}

For any two points $\mathbf{p}(x, 0) \in \mathrm{L} 1, \mathbf{q}(r \cos \theta, r \sin \theta) \in \mathrm{L} 2$, the polar curvature of $\mathbf{p}, \mathbf{q}$ and the origin $\mathbf{o}$ is bounded below by

$$
\begin{aligned}
c_{\mathbf{p}}(\mathbf{o}, \mathbf{p}, \mathbf{q}) & =\operatorname{diam}\{\mathbf{o}, \mathbf{p}, \mathbf{q}\} \cdot \sqrt{\sin ^{2} \theta+\sin ^{2} \angle \mathbf{o p q}+\sin ^{2} \angle \mathbf{o q p}} \\
& \geq \max (x, r) \cdot \sin \theta .
\end{aligned}
$$


Thus, the incidence constant is bounded above by

$$
\begin{aligned}
C_{\mathrm{in}, \mathrm{L}}\left(\mu_{1}, \mu_{2} ; \sigma\right) & =\int_{\mathrm{L} 1} \int_{\mathrm{L} 2} e^{-\frac{c_{\mathrm{p}}(\mathbf{o}, \mathbf{p}, \mathbf{q})}{\sigma}} \mathrm{d} \mu_{1}(\mathbf{p}) \mathrm{d} \mu_{2}(\mathbf{q}) \\
& \leq \int_{0}^{L} \int_{0}^{L} e^{-\frac{\max (x, r) \cdot \sin \theta}{\sigma}} \frac{\mathrm{d} x}{L} \frac{\mathrm{d} r}{L} \\
& =2 \iint_{0 \leq x \leq r \leq L} e^{-\frac{r \sin \theta}{\sigma}} \frac{\mathrm{d} x}{L} \frac{\mathrm{d} r}{L} \\
& =\frac{2}{L} \int_{0}^{L} r \cdot e^{-\frac{r \sin \theta}{\sigma}} \frac{\mathrm{d} r}{L} \\
& =2\left(\frac{\sigma}{L \sin \theta}\right)^{2} \cdot\left(1-e^{-\frac{L \sin \theta}{\sigma}}\left(1+\frac{L \sin \theta}{\sigma}\right)\right) .
\end{aligned}
$$

\section{A.12 Proof of Equation (38) in Example 5.7}

For any $\mathbf{p}\left(x, y_{2}\right) \in \mathrm{R} 1, \mathbf{q}\left(x_{1}, y\right) \in \mathrm{R} 2$, we define $\widetilde{\mathbf{p}}(x, \epsilon) \in \mathrm{R} 1, \widetilde{\mathbf{q}}(\epsilon, y) \in \mathrm{R} 2$. The polar curvature of $\mathbf{p}, \mathbf{q}$ and the origin $\mathbf{o}$ is bounded below by

$$
\begin{aligned}
c_{\mathrm{p}}(\mathbf{o}, \mathbf{p}, \mathbf{q}) & \geq \max (\|\mathbf{o p}\|,\|\mathbf{o q}\|) \cdot \sin \angle \mathbf{p o q} \geq \max (x, y) \cdot \sin \angle \widetilde{\mathbf{p o}} \widetilde{\mathbf{q}} \\
& =\frac{\max (x, y) \cdot\left(x y-\epsilon^{2}\right)}{\sqrt{\left(x^{2}+\epsilon^{2}\right)\left(y^{2}+\epsilon^{2}\right)}} .
\end{aligned}
$$

Thus, the incidence constant is

$$
\begin{aligned}
C_{\mathrm{in}, \mathrm{L}}\left(\mu_{1}, \mu_{2} ; \sigma\right) & =\int_{\mathrm{R} 1} \int_{\mathrm{R} 2} e^{-\frac{c_{\mathrm{p}}(\mathbf{o}, \mathbf{p}, \mathbf{q})}{\sigma}} \frac{\mathrm{d} x}{L} \frac{\mathrm{d} y_{2}}{\epsilon} \frac{\mathrm{d} x_{1}}{\epsilon} \frac{\mathrm{d} y}{L} \\
& \leq \frac{1}{L^{2}} \cdot \int_{\epsilon}^{L+\epsilon} \int_{\epsilon}^{L+\epsilon} e^{-\frac{\max (x, y) \cdot\left(x y-\epsilon^{2}\right)}{\sigma \cdot \sqrt{\left(x^{2}+\epsilon^{2}\right)\left(y^{2}+\epsilon^{2}\right)}}} \mathrm{d} x \mathrm{~d} y .
\end{aligned}
$$

Changing variables $x:=x / \epsilon, y:=y / \epsilon$ and setting $\omega:=L / \epsilon$ gives that

$$
C_{\text {in }}\left(\mu_{1}, \mu_{2} ; \sigma\right) \leq \frac{1}{\omega^{2}} \cdot \int_{1}^{1+\omega} \int_{1}^{1+\omega} e^{-\frac{\max (x, y) \cdot(x y-1)}{\sigma \cdot \sqrt{\left(x^{2}+1\right)\left(y^{2}+1\right)}}} \mathrm{d} x \mathrm{~d} y .
$$

We observe that the integrand is bounded between 0 and 1 , symmetric about $x$ and $y$, and decreasing in each of its arguments. We thus obtain that

$$
\begin{aligned}
C_{\mathrm{in}, \mathrm{L}}\left(\mu_{1}, \mu_{2} ; \sigma\right) \leq & \frac{1}{\omega^{2}} \cdot\left(\int_{1}^{1+\sqrt[4]{\sigma}} \int_{1}^{1+\sqrt[4]{\sigma}}+2 \int_{1}^{1+\sqrt[4]{\sigma}} \int_{1+\sqrt[4]{\sigma}}^{1+\omega}+\int_{1+\sqrt[4]{\sigma}}^{1+\omega} \int_{1+\sqrt[4]{\sigma}}^{1+\omega}\right) \\
\leq & \frac{1}{\omega^{2}} \cdot\left((\sqrt[4]{\sigma})^{2}+2 \cdot \sqrt[4]{\sigma} \cdot(\omega-\sqrt[4]{\sigma}) \cdot e^{\left.-\frac{(1+\sqrt[4]{\sigma}) \cdot(1 \cdot(1+\sqrt[4]{\sigma})-1)}{\left.\sigma \cdot \sqrt{2 \cdot\left(1+\left(1+\sqrt[4]{\left(x^{2}+1\right)\left(y^{2}+1\right)}\right.\right.}\right)}\right)} \mathrm{d} x \mathrm{~d} y\right. \\
& +\frac{1}{\omega^{2}} \cdot(\omega-\sqrt[4]{\sigma})^{2} \cdot e^{-\frac{(1+\sqrt[4]{\sigma}) \cdot\left((1+\sqrt[4]{\sigma})^{2}-1\right)}{\sigma \cdot\left(1+(1+\sqrt[4]{\sigma})^{2}\right)}} \\
\leq & \frac{\sqrt{\sigma}}{\omega^{2}}+\frac{2 \sqrt[4]{\sigma}}{\omega} \cdot e^{-1 /\left(2 \sigma^{3 / 4}\right)}+e^{-1 / \sigma^{3 / 4}} \cdot
\end{aligned}
$$




\section{A.13 Proof of Equation (39) in Example 5.8}

Let $\mathbf{p}(0, \rho \cos \varphi, \rho \sin \varphi) \in \mathrm{D} 1$, and $\mathbf{q}_{1}\left(0, r_{1} \cos \theta_{1}, r_{1} \sin \theta_{1}\right), \mathbf{q}_{2}\left(0, r_{2} \cos \theta_{2}, r_{2} \sin \theta_{2}\right) \in$ D2. Then the polar curvature of these three points and the origin o has the following lower bound:

$$
c_{\mathrm{p}}\left(\mathbf{o}, \mathbf{p}, \mathbf{q}_{1}, \mathbf{q}_{2}\right) \geq|\mathbf{o p}| \cdot \operatorname{psin}_{\mathbf{o}}\left(\mathbf{p}, \mathbf{q}_{1}, \mathbf{q}_{2}\right)=\rho \cdot \sin \varphi \sin \left|\theta_{1}-\theta_{2}\right| .
$$

Due to the symmetry of the two disks, we have that

$$
\begin{aligned}
C_{\mathrm{in}, \mathrm{L}}\left(\mu_{1}, \mu_{2} ; \sigma\right) & =\int_{\mathrm{D} 1} \int_{\mathrm{D} 2} \int_{\mathrm{D} 2} e^{-c_{\mathrm{p}}\left(\mathbf{o}, \mathbf{p}, \mathbf{q}_{1}, \mathbf{q}_{2}\right) / \sigma} \mathrm{d} \mu_{1}(\mathbf{p}) \mathrm{d} \mu_{2}\left(\mathbf{q}_{1}\right) \mathrm{d} \mu_{2}\left(\mathbf{q}_{2}\right) \\
& \leq \int_{0}^{1} \int_{0}^{\pi} \int_{-\pi / 2}^{\pi / 2} \int_{-\pi / 2}^{\pi / 2} e^{-\frac{\rho \sin \varphi \cdot \sin \left|\theta_{1}-\theta_{2}\right|}{\sigma} \frac{\rho \mathrm{d} \rho \mathrm{d} \varphi}{\pi / 2} \frac{\mathrm{d} \theta_{1}}{\pi} \frac{\mathrm{d} \theta_{2}}{\pi}} \\
& =\frac{4}{\pi^{3}} \cdot \int_{0}^{1} \int_{0}^{\pi} \iint_{-\frac{\pi}{2} \leq \theta_{2} \leq \theta_{1} \leq \frac{\pi}{2}}^{e^{\frac{-\rho \sin \varphi \cdot \sin \left(\theta_{1}-\theta_{2}\right)}{\sigma}} \rho \mathrm{d} \rho \mathrm{d} \varphi \mathrm{d} \theta_{1} \mathrm{~d} \theta_{2} .}
\end{aligned}
$$

Changing variables $\theta:=\theta_{1}-\theta_{2}, \theta_{2}:=\theta_{2}$ and exchanging the corresponding double integral, we obtain that

$$
\begin{aligned}
C_{\mathrm{in}, \mathrm{L}}\left(\mu_{1}, \mu_{2} ; \sigma\right) & \leq \frac{4}{\pi^{3}} \cdot \int_{0}^{1} \int_{0}^{\pi} \int_{0}^{\pi} e^{-\frac{\rho \sin \varphi \cdot \sin \theta}{\sigma}} \rho \mathrm{d} \rho \mathrm{d} \varphi(\pi-\theta) \mathrm{d} \theta \\
& \leq \frac{4}{\pi^{2}} \cdot \int_{0}^{1} \int_{0}^{\pi} \int_{0}^{\pi} e^{-\frac{\rho \sin \varphi \cdot \sin \theta}{\sigma}} \rho \mathrm{d} \rho \mathrm{d} \varphi \mathrm{d} \theta \\
& =\frac{16}{\pi^{2}} \cdot \int_{0}^{1} \int_{0}^{\pi / 2} \int_{0}^{\pi / 2} e^{-\frac{\rho \sin \varphi \cdot \sin \theta}{\sigma}} \rho \mathrm{d} \rho \mathrm{d} \varphi \mathrm{d} \theta .
\end{aligned}
$$

We observe that the integrand is bounded between 0 and 1 , symmetric about $\varphi$ and $\theta$, and decreasing in each of them. Thus,

$$
\begin{aligned}
C_{\mathrm{in}, \mathrm{L}}\left(\mu_{1}, \mu_{2} ; \sigma\right) \leq & \frac{16}{\pi^{2}} \cdot \int_{0}^{1}\left(\int_{0}^{\sqrt[4]{\sigma}} \int_{0}^{\sqrt[4]{\sigma}}+2 \int_{0}^{\sqrt[4]{\sigma}} \int_{\sqrt[4]{\sigma}}^{\frac{\pi}{2}}+\int_{\sqrt[4]{\sigma}}^{\frac{\pi}{2}} \int_{\sqrt[4]{\sigma}}^{\frac{\pi}{2}}\right) \\
\leq & \frac{16}{\pi^{2}} \cdot\left((\sqrt[4]{\sigma})^{2}+2 \cdot \sqrt[4]{\sigma} \cdot\left(\frac{\pi}{2}-\sqrt[4]{\sigma}\right)\right) \cdot \int_{0}^{1} \rho \mathrm{d} \rho \\
& +\frac{16}{\pi^{2}} \cdot\left(\frac{\pi}{2}-\sqrt[4]{\sigma}\right)^{2} \cdot \int_{0}^{1} e^{\left.-\frac{\rho \cdot(\sin \theta}{\sigma} \sqrt[4]{\sigma}\right)^{2}} \rho \mathrm{d} \rho \\
\leq & \frac{8 \sqrt{\sigma}}{\pi^{2}}+\frac{8 \sqrt[4]{\sigma}}{\pi}+\frac{4 \sigma^{2}}{(\sin \sqrt[4]{\sigma})^{4}}
\end{aligned}
$$

\section{References}

[1] S. Agarwal, K. Branson, and S. Belongie. Higher order learning with graphs. In Proceedings of the 23rd International Conference on Machine learning, volume 148, pages 17-24, 2006.

[2] S. Agarwal, J. Lim, L. Zelnik-Manor, P. Perona, D. Kriegman, and S. Belongie. Beyond pairwise clustering. In Proceedings of the 2005 IEEE Computer Society Conference on Computer Vision and Pattern Recognition (CVPR'05), volume 2, pages 838-845, 2005. 
[3] E. Arias-Castro, D. Donoho, and X. Huo. Near-optimal detection of geometric objects by fast multiscale methods. IEEE Transactions on Information Theory, 51(7), July 2005.

[4] B. Bader and T. Kolda. Matlab tensor classes for fast algorithm prototyping. Technical Report SAND2004-5187, Sandia National Laboratories, October 2004.

[5] P. Bradley and O. Mangasarian. k-plane clustering. J. Global optim., 16(1):23-32, 2000.

[6] M. Brand and K. Huang. A unifying theorem for spectral embedding and clustering. In Proceedings of the Ninth International Workshop on Artificial Intelligence and Statistics, January 2003.

[7] G. Chen and G. Lerman. Spectral curvature clustering (SCC). Int. J. Comput. Vision, 81(3):317-330, 2009.

[8] J. Costeira and T. Kanade. A multibody factorization method for independently moving objects. International Journal of Computer Vision, 29(3):159-179, 1998.

[9] M. Fischler and R. Bolles. Random sample consensus: A paradigm for model fitting with applications to image analysis and automated cartography. Comm. of the ACM, 24(6):381-395, June 1981.

[10] G. Golub and C. Van Loan. Matrix Computations. John Hopkins University Press, Baltimore, Maryland, 1996.

[11] V. Govindu. A tensor decomposition for geometric grouping and segmentation. In CVPR, volume 1, pages 1150-1157, June 2005.

[12] P. Gruber and F. Theis. Grassmann clustering. In Proc. EUSIPCO 2006, Florence, Italy, 2006.

[13] G. Haro, G. Randall, and G. Sapiro. Translated poisson mixture model for stratification learning. Int. J. Comput. Vision, 80(3):358-374, 2008.

[14] J. Ho, M. Yang, J. Lim, K. Lee, and D. Kriegman. Clustering appearances of objects under varying illumination conditions. In Proceedings of International Conference on Computer Vision and Pattern Recognition, volume 1, pages $11-18,2003$.

[15] A. Hyvärinen and E. Oja. Independent component analysis: algorithms and applications. Neural Netw., 13(4-5):411-430, 2000.

[16] A. Kambhatla and T. Leen. Fast non-linear dimension reduction. In $A d-$ vances in Neural Information Processing Systems 6, pages 152-159, 1994.

[17] K. Kanatani. Motion segmentation by subspace separation and model selection. In Proc. of 8th ICCV, volume 3, pages 586-591. Vancouver, Canada, 2001.

[18] K. Kanatani. Evaluation and selection of models for motion segmentation. In rth ECCV, volume 3, pages 335-349, May 2002. 
[19] D. Kushnir, M. Galun, and A. Brandt. Fast multiscale clustering and manifold identification. Pattern Recognition, 39(10):1876-1891, October 2006.

[20] L. De Lathauwer, B. De Moor, and J. Vandewalle. A multilinear singular value decomposition. SIAM J. Matrix Anal. A., 21(4):1253-1278, 2000.

[21] G. Lerman and J. T. Whitehouse. High-dimensional Menger-type curvatures - part I: Geometric multipoles and multiscale inequalities. Submitted. Available from http://arxiv.org/abs/0805.1425v1.

[22] G. Lerman and J. T. Whitehouse. High-dimensional Menger-type curvatures - part II: $d$-separation and a menagerie of curvatures. Submitted. Available from http://arxiv.org/abs/0809.0137v1.

[23] G. Lerman and J. T. Whitehouse. Least squares for probability measures via multi-way curvatures. In preparation.

[24] G. Lerman and J. T. Whitehouse. On $d$-dimensional $d$ semimetrics and simplex-type inequalities for high-dimensional sine functions. Journal of Approximation Theory (2008), http://dx.doi.org/10.1016/j.jat.2008.03.005, 2008.

[25] Y. Ma, H. Derksen, W. Hong, and J. Wright. Segmentation of multivariate mixed data via lossy coding and compression. IEEE Transactions on Pattern Analysis and Machine Intelligence, 29(9):1546-1562, September 2007.

[26] Y. Ma, A. Y. Yang, H. Derksen, and R. Fossum. Estimation of subspace arrangements with applications in modeling and segmenting mixed data. SIAM Review, 50(3):413-458, 2008.

[27] J. MacQueen. Some methods for classification and analysis of multivariate observations. In Proceedings of the 5th Berkeley Symposium on Mathematical Statistics and Probability, volume 1, pages 281-297. University of California Press, Berkeley, CA, 1967.

[28] J. Mairal, F. Bach, J. Ponce, G. Sapiro, and A. Zisserman. Discriminative learned dictionaries for local image analysis. In Proc. CVPR, Alaska, June 2008.

[29] C. McDiarmid. On the method of bounded differences. In Surveys in combinatorics, pages 148-188. Cambridge University Press, 1989

[30] G. Medioni, M.-S. Lee, and C.-K. Tang. A Computational Framework for Segmentation and Grouping. Elsevier, 2000.

[31] A. Ng, M. Jordan, and Y. Weiss. On spectral clustering: Analysis and an algorithm. In Advances in Neural Information Processing Systems 14, pages 849-856, 2001.

[32] A. Shashua, R. Zass, and T. Hazan. Multi-way clustering using supersymmetric non-negative tensor factorization. In ECCV06, volume IV, pages 595-608, 2006. 
[33] J. Shi and J. Malik. Normalized cuts and image segmentation. IEEE Transactions on Pattern Analysis and Machine Intelligence, 22(8):888-905, August 2000.

[34] R. Souvenir and R. Pless. Manifold clustering. In the 10th International Conference on Computer Vision (ICCV 2005), 2005.

[35] A. Szlam. Modifications of $k q$-flats for supervised learning. 2008.

[36] M. Tipping and C. Bishop. Mixtures of probabilistic principal component analysers. Neural Computation, 11(2):443-482, 1999.

[37] P. H. S. Torr. Geometric motion segmentation and model selection. Phil. Trans. R. Soc. Lond. A, 356:1321-1340, 1998.

[38] P. Tseng. Nearest $q$-flat to $m$ points. Journal of Optimization Theory and Applications, 105(1):249-252, April 2000.

[39] R. Vidal, Y. Ma, and S. Sastry. Generalized principal component analysis (GPCA). IEEE Transactions on Pattern Analysis and Machine Intelligence, $27(12), 2005$.

[40] U. von Luxburg, M. Belkin, and O. Bousquet. Consistency of spectral clustering. The Annals of Statistics, 36(2):555-586, 2008.

[41] J. Yan and M. Pollefeys. A general framework for motion segmentation: Independent, articulated, rigid, non-rigid, degenerate and nondegenerate. In $E C C V$, volume 4, pages 94-106, 2006.

[42] A. Y. Yang, S. R. Rao, and Y. Ma. Robust statistical estimation and segmentation of multiple subspaces. In Computer Vision and Pattern Recognition Workshop, June 2006.

[43] L. Zwald and G. Blanchard. On the convergence of eigenspaces in kernel principal components analysis. In Advances in Neural Information Processing Systems 18, pages 1649-1656, 2005. 